This deposited item is the version of the article (RSC Advances, 2016, DOI: 10.1039/C6RA07206B) accepted for publication in RSC Advance on April 25, 2016. 


\title{
Efficient, Robust Surface Functionalization and Stabilization of Gold Nanorods with Quaternary Ammonium-Containing Ionomers as Multidentate Macromolecular Ligands**
}

\author{
Zhongmin Dong, Peng Xiang, Lingqi Huang, and Zhibin Ye
}

Bharti School of Engineering, Laurentian University, 935 Ramsey Lake Road, Sudbury, Ontario P3E 2C6, Canada

* Corresponding author; email: zye@laurentian.ca

Keywords: gold nanorods, surface modification, ionomers, ligand exchange, nanocomposites

\begin{abstract}
Surface functionalization of gold nanorods (GNRs) is critical to their applications in various fields. While there are several existing strategies, we report in this article a new general strategy for the surface functionalization of GNRs with quaternary ammonium-containing ionomers as a novel class of multidentate macromolecular surface ligands. A range of tetralkylammoniumcontaining hyperbranched polyethylene- and linear poly(n-butyl acrylate)-based ionomers has been specifically designed and employed in the strategy. Acting as multidentate macromolecular analogues of cetyltrimethylammonium bromide (CTAB), the ionomers have been demonstrated to bind onto the GNR surface by displacing the surface-bound CTAB species via ligand exchange to render CTAB-free ionomer-modified GNRs. By properly designing the enabling ionomers, we have shown that the modified GNRs can be endowed with some desired properties, such as excellent dispersibility in various organic solvents, robust stability under multiple rounds (up to 12 investigated) of high-speed centrifugation in organic solvents, amphiphilicity with dispersibility in both aqueous and organic media, fluorescence, and capability in carrying hydrophobic guest species. This strategy thus provides potential new ways for the construction of novel multifunctional GNR nanocomposites.
\end{abstract}




\section{Introduction}

Due to their intriguing, tunable optical properties, gold nanorods (GNRs) as a unique class of anisotropic metallic nanoparticles have attracted enormous research interest for broad applications in sensing, biomedical imaging, photothermal cancer therapy, drug delivery, functional polymer nanocomposites, etc. ${ }^{1-10}$ GNRs are commonly synthesized using the convenient seed-mediated growth methodology developed by Murphy and El-Sayed. ${ }^{11-14}$ This wet chemical synthesis requires the use of cetyltrimethylammonium bromide (CTAB) as both the shape-directing agent and the surface stabilizing ligand. As-prepared GNRs are non-covalently coated with a zipper-like CTAB bilayer, where the cationic quaternary ammonium head groups in the inner layer bind strongly to the rod surface and the head groups in the outer layer protruding out to provide the stability of GNRs in the aqueous solution. ${ }^{15}$ It is known that there is a constant dynamic exchange of CTAB molecules between the solution and rod surface, and the concentration of free $\mathrm{CTAB}$ in the aqueous solution must be maintained above a certain value for the CTAB-coated GNRs to remain soluble. ${ }^{16}$ Because of this feature, the as-prepared CTABcoated GNRs are often not directly usable for many applications. For biological applications, their use is particularly prohibited since free CTAB has been known to be highly cytotoxic. ${ }^{17}$ For functional polymer nanocomposite applications, CTAB-coated GNRs are generally immiscible with the matrix polymer and aggregate upon mixing. ${ }^{10}$ In addition, CTAB-coated GNRs, though soluble/stable in aqueous solution containing sufficient free CTAB, tend to quickly aggregate in most organic solvents due to the destabilization of the CTAB bilayer, while their solubility in organic media is highly desired in order for further functionalization with organic functional groups to fine-tune their properties. ${ }^{18}$ As such, surface functionalization of the GNRs by displacing $\mathrm{CTAB}$ with another proper surface ligand via ligand exchange has been crucial in most applications in order to render their stability at the required condition/media as well as to provide added functionality. $3,5,6,10,18,19$

To date, four common strategies have been employed for the surface functionalization of

GNRs. ${ }^{3,5,6,10,18,19}$ The most common one employs the classic gold-thiol bond chemistry. A class of sulfur-containing molecules (having thiol, disulfide, or dithiocarbamate group) has been devised for this purpose, ${ }^{3,5,6,10,18,19}$ such as thiolated polyethylene glycol (PEG), ${ }^{20-22}$ (16- 
mercaptohexadecyl)trimethylammonium bromide (a thiolated CTAB analogue), ${ }^{23}$ alkyl thiol acids/esters, ${ }^{24-26}$ and thiolated DNA. ${ }^{27,28}$ These sulfur-containing molecules can bind strongly to the rod surface by forming the covalent $\mathrm{Au}-\mathrm{S}$ bond. However, in the case with most small sulfurcontaining molecules, their simple addition may not lead to complete functionalization and the presence of additional thiolated PEG is often required in order to prevent rod aggregation. ${ }^{6,26-29}$ An alternative strategy utilizes the layer-by-layer deposition of polyelectrolytes directly onto the CTAB-coated GNRs through electrostatic interactions. This strategy facilitates the convenient introduction of chemically reactive groups in the polyelectrolyte coating for tethering of various functional species..$^{30-34}$ Thirdly, a surfactant exchange strategy has been employed to partially displace $\mathrm{CTAB}$ on rod surface with the use of small molecule quaternary ammonium-containing CTAB analogues, such as phospholipids ${ }^{16,35,36}$ and a polymerizable cationic surfactant. ${ }^{37}$ Lastly, $^{2}$ GNRs may also be coated with a silica shell, followed with subsequent attachment of desired functional molecules. ${ }^{38-40}$ These strategies have facilitated the synthesis of a large family of surface-modified/functionalized GNRs suiting various specific applications. ${ }^{3-10}$

We have been on the search for alternative surface functionalization strategies that will further expand the toolbox of functional surface ligands and provide unique opportunities for the construction of novel multifunctional GNR nanocomposites of new/improved properties. In this article, we demonstrate for the first time the design and use of quaternary ammonium-containing ionomers as a new class of surface ligands for the efficient stabilization and functionalization of GNRs by convenient ligand exchange. Ionomers are polymers containing a small fraction of ionic functional groups (typically less than $10 \mathrm{~mol} \%$ ) that are covalently bonded to the polymer backbone as pendant groups. ${ }^{41}$ Though polymers containing ionic groups (such as imidazolium groups) have been employed for the tethering and/or stabilization of small gold nanoparticles, ${ }^{42-44}$ ionomers have not yet been reported for the functionalization of GNRs having significantly larger sizes. We have herein tailor-designed a range of hyperbranched polyethylene- and linear poly(n-butyl acrylate)-based ionomers containing covalently tethered cationic tetralkylammonium ions as well as other optional functionalities (such as fluorescent pyrene groups, amphiphilic biocompatible oligo(ethylene glycol) (OEG) side blocks, or initiating sites of living radical polymerization). Acting as the multidentate macromolecular CTAB analogues, these iononmers has been found to strongly bind onto GNR surface through their multidentate 
quaternary ammonium groups via ligand exchange. As such, the ionomers can facilitate efficient phase transfer of GNRs from aqueous phase to various nonpolar or low-polarity organic solvents. The resulting modified GNRs can robustly sustain multiple (as high as 12 investigated herein) rounds of high-speed centrifugation (at 11,176 $\mathrm{g}$ for $20 \mathrm{~min}$ ) without irreversible aggregations. This consequently enables the thorough displacement of CTAB. Meanwhile, the functionalized GNRs can be stably dispersed in a range of organic solvents with varying polarity. In particular, with the use of amphiphilic ionomers containing OEG side blocks, the functionalized GNRs can be stably dispersed in both aqueous and organic phases. In addition, by building designed functional groups into the ionomers, we also demonstrate that the resulting modified GNRs can be endowed with functional properties, such as fluorescence and carrier of hydrophobic guest species. This ionomer functionalization method can thus be employed as a stepping-stone for creating new multifunctional GNR nanocomposites.

\section{Results and Discussion}

\section{Ionomer Design and Synthesis of CTAB-Coated GNRS}

Our design of the quaternary ammonium-containing ionomers for the functionalization of GNRs is inspired by the strong binding of CTAB inner layer to the rod surface in the CTAB-coated GNRs. Though generally considered weaker than the covalent $\mathrm{Au}-\mathrm{S}$ bond, the binding of the

cationic $\mathrm{CTAB}$ head group to rod surface has been reported to be stable even at $350{ }^{\circ} \mathrm{C} .{ }^{15}$ With this, we hypothesized that ionomers containing multiple quaternary ammonium groups per chain should also strongly adsorb onto GNRs through the multidentate binding of their quaternary ammonium groups to the nanorod surface as multidentate macromolecular analogues of CTAB, which can consequently be used to functionalize GNRs by displacing the CTAB bilayer.

With an initial intent to mimic the hydrocarbon tail of CTAB, we first chose to design quaternary ammonium-containing hydrocarbon polyethylene-based ionomers. Considering that linear polyethylene with long straight ethylene sequences are semicrystalline with poor solvent solubility at ambient temperature, we rationalized to design hyperbranched polyethylene ionomers (HPEIs), whose skeleton is constructed with hyperbranched ethylene sequences. 
Featured with extensive branch-on-branch structures, the hyperbranched skeleton makes the ionomers completely amorphous and dispersible/soluble in many nonpolar and low-polarity solvents at room temperature. ${ }^{45,46} \mathrm{We}$ expected that the resulting modified GNRs would thus inherit the dispersibility in these solvents. A range of HPEIs (HPEI0-HPEI6) have thus been synthesized herein by $\mathrm{Pd}$-diimine-catalyzed chain walking copolymerization of ethylene with an acrylate-type ionic liquid comonomer having a quaternary ammonium ion with $\mathrm{BF}_{4}$ counter anion ([2-(acryloyloxy)ethyl]trimethylammonium tetrafluoroborate $\left(\mathrm{AETA}^{+} \mathrm{BF}_{4}^{-}\right)$, see Scheme 1a). Other optional functional acrylate comonomers (see Scheme 1a) were also used to introduce additional functionalities into the ionomers, including pyrenemethyl acrylate (PMA) containing a fluorescent pyrene group, oligo(ethylene glycol) methyl ether acrylate (OEGA) containing a short OEG block, 2-(2-bromoisobutyryloxy)ethyl acrylate (BIEA) containing a bromoisobutyryl initiating site. ${ }^{47}$ Following the polymerization mechanism, ${ }^{45,46}$ all the ionomers should be random copolymers with each chain containing multiple quaternary ammonium groups on average. See Tables 1 and S1 in ESI for their synthesis and macromolecular structural details, and Figure S1 in ESI for representative ${ }^{1} \mathrm{H}$ NMR spectra. After the polymerization, all the ionomers underwent anion exchange by replacing the $\mathrm{BF}_{4}^{-}$counter anion with $\mathrm{Cl}^{-} . \mathrm{Pd}$-diimine-catalyzed chain walking polymerization is well known for their unique capability in rendering hyperbranched polyethylenes as well as their functionalized analogues by copolymerization of ethylene with acrylate comonomers. ${ }^{45}$ The highly compact dendrimer-like hyperbranched polymer skeleton results from the characteristic chain walking mechanism of the Pd-diimine catalysts. ${ }^{45,46} \mathrm{We}$ have previously synthesized a large class of such polymers ${ }^{45,46,48-54}$ and have recently demonstrated, in particular, the synthesis of hyperbranched polyethylene ionomers by direct copolymerization of ethylene with acrylate-type quaternary ammonium-containing ionic liquid comonomers. ${ }^{55}$

To demonstrate that this ionomer functionalization strategy is general and not just limited to the hyperbranched polyethylene-based ionomers, we have also synthesized a quaternary ammoniumcontaining, linear poly( $n$-butyl acrylate)-based ionomer (PBAI) by free radical terpolymerization of $n$-butyl acrylate (BA), $\mathrm{AETA}^{+} \mathrm{BF}_{4}^{-}$, and PMA (see Scheme 1b). As per ${ }^{1} \mathrm{H}$ NMR spectroscopy (see Figure S2 in ESI), PBAI has a quaternary ammonium content of $2.0 \mathrm{~mol} \%$ and PMA content of $0.3 \mathrm{~mol} \%$. 
(a)

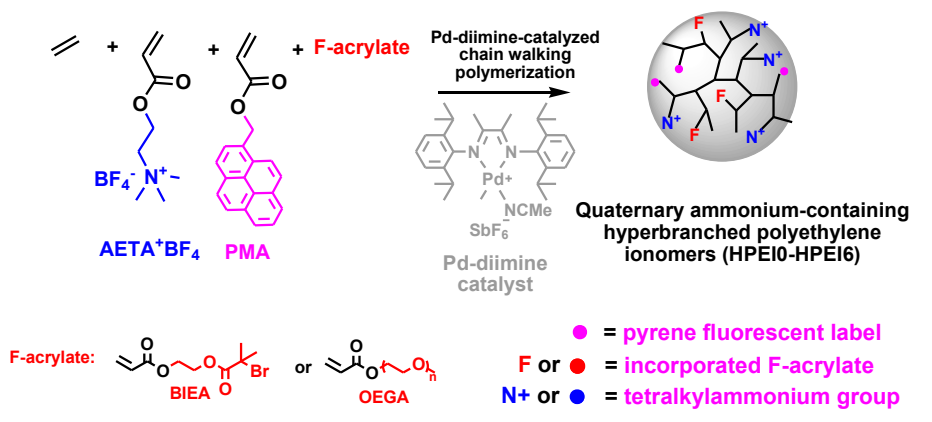

(b)
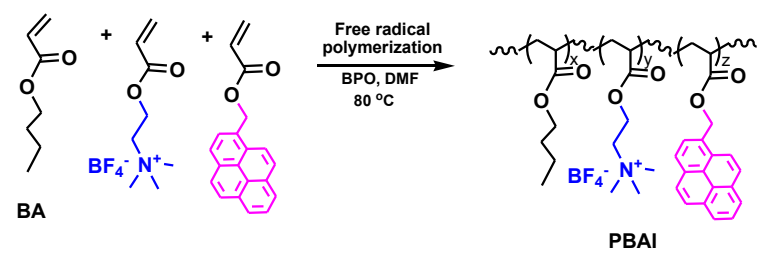

Scheme 1. Synthesis of (a) hyperbranched polyethylene ionomers (HPEI0-HPEI6) by Pddiimine-catalyzed chain walking polymerization and (b) linear poly( $n$-butyl acrylate) ionomer (PBAI) by radical polymerization.

Table 1. Quaternary ammonium-containing hyperbranched polyethylene ionomers (HPEIs) synthesized via Pd-diimine-catalyzed chain walking copolymerization. ${ }^{a}$

\begin{tabular}{|c|c|c|c|c|c|c|c|c|c|}
\hline \multirow[b]{2}{*}{ ionomer } & \multicolumn{4}{|c|}{ comonomer feed concentration } & \multicolumn{3}{|c|}{ ionomer composition $(\mathrm{mol} \%)^{c}$} & \multirow{2}{*}{$\begin{array}{c}M_{\mathrm{w}}{ }^{d} \\
(\mathrm{kDa})\end{array}$} & \multirow[b]{2}{*}{$\mathrm{PDI}^{d}$} \\
\hline & $\begin{array}{c}\mathrm{AETA}^{+} \mathrm{BF}_{4}^{-} \\
\text {(M) }\end{array}$ & $\begin{array}{l}\text { PMA } \\
\text { (M) }\end{array}$ & $\begin{array}{c}\text { F-acrylate }^{b} \\
\text { (M) }\end{array}$ & $\mathrm{F}$ & $\mathrm{AETA}^{+}$ & PMA & F-acrylate & & \\
\hline $\mathrm{HPEI0}^{e}$ & 0.3 & - & - & - & 1.2 & - & - & 18.8 & 1.42 \\
\hline HPEI $1^{f}$ & 0.2 & 0.03 & - & - & 0.81 & 0.07 & - & 16.3 & 1.33 \\
\hline HPEI2 & 0.1 & 0.10 & 0.1 & $\mathrm{Br}$ & 0.15 & 0.63 & 0.25 & 43.3 & 1.13 \\
\hline HPEI3 & 0.2 & 0.03 & 0.08 & $\mathrm{Br}$ & 0.28 & 0.22 & 0.27 & 8.8 & 2.08 \\
\hline HPEI4 & 0.5 & 0.03 & 0.24 & $\mathrm{Br}$ & 1.0 & 0.41 & 1.0 & 7.8 & 2.29 \\
\hline HPEI5 & 0.6 & 0.03 & 0.4 & $\mathrm{Br}$ & 1.8 & 0.27 & 1.6 & 11.0 & 1.26 \\
\hline HPEI6 & 0.8 & 0.03 & 0.8 & OEG & 1.7 & 0.34 & 11 & 8.3 & 2.69 \\
\hline
\end{tabular}

${ }^{a}$ Other copolymerization conditions: solvent, acetone $(10 \mathrm{~mL})$; ethylene pressure, 1 atm; room temperature; $\mathrm{Pd}$-diimine catalyst, $0.1 \mathrm{mmol} .{ }^{b}$ F-acrylate represents $\mathrm{BIEA}$ with active $\mathrm{Br}$ functionality $(\mathrm{F}=\mathrm{Br})$ or OEGA with a OEG block $(\mathrm{F}=\mathrm{OEG}) .{ }^{c}$ Molar content of the acrylate comonomers in the ionomers determined with ${ }^{1} \mathrm{H}$ NMR spectroscopy. ${ }^{d}$ The weight-average molecular weight $\left(M_{\mathrm{w}}\right)$ and polydispersity index (PDI) determined with gel permeation chromatography (GPC). ${ }^{e}$ The copolymer of ethylene with $\mathrm{AETA}^{+} \mathrm{BF}_{4}^{-}$alone. ${ }^{f}$ The terpolymer of ethylene with $\mathrm{AETA}^{+} \mathrm{BF}_{4}^{-}$and PMA.

For this investigation, two large-scale batches of CTAB-coated GNRs (termed subsequently as long GNRs and short GNRs) with different aspect ratios (6.0 and 4.7, respectively, with the same 
nanorod diameter of $7.4 \mathrm{~nm}$ ) were synthesized by scaling up the seed-mediated growth methods reported by Zubarev ${ }^{56}$ and El-Sayed, ${ }^{14}$ respectively, to $800 \mathrm{~mL}$. Figure 1 shows their TEM images, along with their UV-vis absorbance spectra in water. UV-vis spectra show their longitudinal surface plasmon resonance (LSPR) band maxima $\left(\lambda_{\text {LSPR }}\right)$ at 983 and $840 \mathrm{~nm}$, respectively, while with the identical transverse surface plasmon resonance (TSPR) band $\operatorname{maximum}\left(\lambda_{\mathrm{TSPR}}\right)$ at $510 \mathrm{~nm}$.
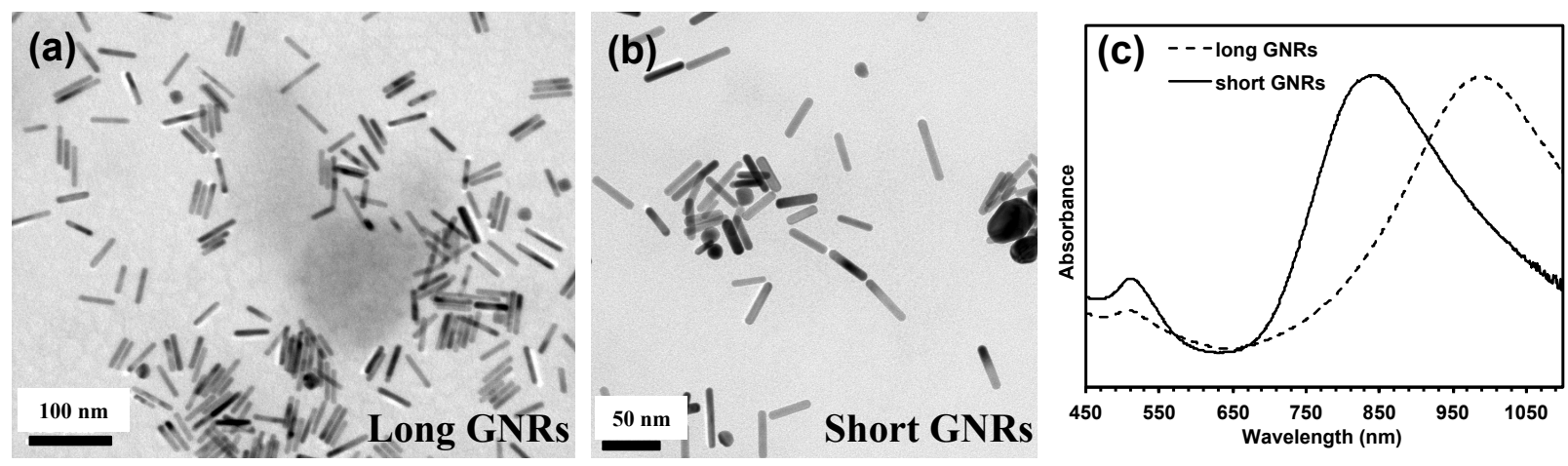

Figure 1. TEM images for (a) CTAB-coated long GNRs (average length, $44 \pm 5 \mathrm{~nm}$; average diameter, $7.4 \pm 0.8 \mathrm{~nm}$; average aspect ratio, 6.0; measured on more than 100 nanorods) and (b) CTAB-coated short GNRs (average length, $35 \pm 7 \mathrm{~nm}$; average diameter, $7.4 \pm 0.8 \mathrm{~nm}$; average aspect ratio, 4.7; measured on more than 100 nanorods); (c) their UV-vis spectra in aqueous solution (normalized with respect to their LSPR peaks).

\section{Phase Transfer of GNRs from Aqueous Phase to Organic Phases with HPEIO}

We discovered that convenient phase transfer of GNRs from aqueous phase to various waterimmiscible nonpolar or low-polarity organic phases (including chlorobenzene, chloroform, toluene, and hexane) can be facilitated with the use of HPEIO. HPEI0, designed as a copolymer of ethylene and $\mathrm{AETA}^{+} \mathrm{BF}_{4}^{-}$, contains solely quaternary ammonium ions at $1.2 \mathrm{~mol} \%$ while with no other functionalities. It dissolves well in nonpolar or low-polarity solvents to which the hyperbranched polyethylene skeleton has good affinity, but not in aqueous phase or high-polarity solvents. To enable the phase transfer, the dark-colored aqueous phase of long GNRs (2 mL with $[\mathrm{Au}]=2.0 \mathrm{mg} / \mathrm{mL}$ ) was placed together with the respective colorless organic phase containing dissolved HPEI0 (2 mL with [HPEI0] $=5 \mathrm{mg} / \mathrm{mL}$ ) (see the upper photographs in the inset of 
Figure 2 for the biphase mixtures prior to phase transfer). Following a thorough mixing, the twophase mixtures all turned into emulsion with no clear phase separation due to the presence of free CTAB. Upon the addition of a small amount of $\mathrm{NaCl}$ as the demulsifier, phase separation was reestablished immediately, along with the swift relocation of GNRs into the organic phases under gentle stirring. As shown in the lower photographs in the inset in Figure 2, the organic phases all turned dark with no precipitates while the aqueous phases became almost colorless following the phase transfer, indicating the nearly complete transfer of GNRs from the aqueous phase to organic phase. Moreover, the two-phase systems were found stable at least 3 months after the phase transfer.

The UV-vis spectra of the various organic phases (after the same dilution) following the phase transfer are shown in Figure 2. The characteristic LSPR band of GNRs is well retained following the phase transfer. It shows an increasing red shift relative to that of CTAB-coated long GNRs $\left(\lambda_{\text {LSPR }}=983 \mathrm{~nm}\right)$ with the increase in the refractive index of the solvents $\left(\lambda_{\text {LSPR }}=1022,1033\right.$, 1037, and $1057 \mathrm{~nm}$ in hexane, toluene, chloroform, and chlorobenzene, respectively), due to its very high sensitivity to the refractive index of the surrounding environment. ${ }^{3,6,9,57-61}$ In addition, the intensity of the LSRP band after normalization with respect to the TSRP band also shows small but noticeable enhancements in chloroform, toluene, and chlorobenzene, which should also result from the pronounced increases in solvent refractive index. ${ }^{61}$ These UV-vis spectra thus confirm the successful transfer of GNRs from the aqueous phase to the various organic phases in the presence of HPEIO as the phase transfer agent. 


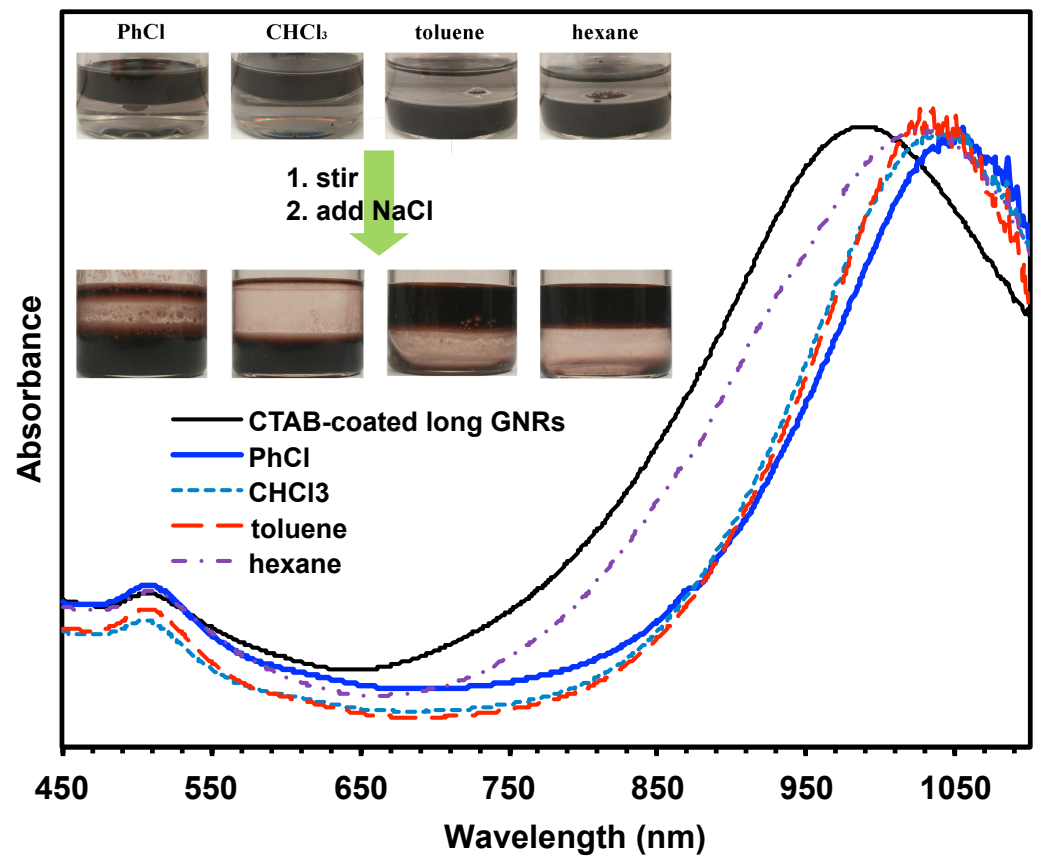

Figure 2. Phase transfer of CTAB-coated long GNRs from aqueous phase to four nonpolar or low-polarity organic phases, chlorobenzene $(\mathrm{PhCl})$, chloroform, toluene, and hexane, containing HPEI0: UV-vis absorbance spectra of the resulting organic phases following the phase transfer (normalized with respect to their LSPR bands), along with that of CTAB-coated long GNRs in aqueous phase for comparison. The inset shows the photographs of the biphase mixtures before and after phase transfer.

Control experiments were also performed in toluene as the organic phase under otherwise identical conditions but in the presence of a nonionic hyperbranched polyethylene homopolymer (HPE) without containing quaternary ammonium ions (synthesized by ethylene homopolymerization $)^{55}$ or in the absence of any polymer. The inset in Figure S4 in ESI shows the photographs of the control experiments both before and after the same mixing and salt addition procedure. Following the procedure, the aqueous phase in both cases remained very dark while the color of organic phases were only slightly darkened with negligible presence of GNRs according to their UV-vis spectra (Figure S4), indicating the unsuccessful phase transfer. These control experiments thus confirm the critical role of the quaternary ammonium-containing ionomer in facilitating the phase transfer. We envisage that the addition of the salt into the aqueous phase dispels the GNRs from the aqueous phase to the organic phase, where the desorption of the CTAB bilayer occurs due to the drastically enhanced critical micelle 
concentration of CTAB in the organic phase. This is accompanied with the multidentate binding of the ionomer onto the GNR surface by displacing some of the bound CTAB ligands renders an ionomer layer coating the GNRs. Because of the high affinity of the hyperbranched polyethylene skeleton in the adsorbed ionomer to nonpolar or low-polarity solvents, the resulting ionomermodified GNRs can thus be stably dispersed in the organic phases. Meanwhile, the ionomer coating layer also acts as a barrier layer effectively preventing their aggregation.

Several earlier studies have shown the phase transfer of GNRs from aqueous phase to organic phases including chloroform, ${ }^{62,63}$ dichloromethane, ${ }^{64,65}$ an ionic liquid, ${ }^{66}$ or a alkanethiol/acetone mixture. ${ }^{67}$ Those earlier reports often require the covalent surface modification of GNRs with thiolated PEG or other thiolated molecules or need special solvents (i.e., ionic liquid). The stable dispersion of GNRs in the common nonpolar or low-polarity solvents facilitated noncovalently with the ionomers can thus provide new opportunities for their further functionalization required to be done in these media.

\section{Preparation of CTAB Free, Ionomer-Modified GNRs with Dispersibility in Organic Solvents by Direct Dropping Method}

After confirming the binding capability of the ionomers to the GNR surface in the above phase transfer experiments, we have subsequently developed a simple direct dropping method to conveniently prepare CTAB-free ionomer-modified GNRs with dispersibility in organic solvents, and to quantitatively study the adsorption of ionomers on GNR surface. To facilitate the quantitative study, we used particularly the range of pyrene-labeled HPEIs (HPEI1-HPEI5), which can dissolve well in nonpolar or low-polarity solvents.

In the direct dropping method, the concentrated aqueous dispersion of CTAB-coated GNRs at a prescribed volume is dropped directly, under vigorous stirring, into an ionomer solution (HPEI1HPEI5) in tetrahydrofuran (THF) as a water-miscible solvent. Therein, the volume of the aqueous solution is maintained low relative to the organic ionomer solution to avoid the possible precipitation of the ionomers upon the addition of the aqueous phase. The feed mass ratio of ionomer to GNRs $\left[\left(m_{\text {ionomer }} / m_{\mathrm{Au}}\right)_{0}\right]$ is generally maintained above $\geq 0.9$ (see Table 2$)$ to ensure the 
presence of excess ionomer for coating the GNRs. It was found that immediate severe aggregation occurs when the aqueous GNR dispersion is dropped into pure THF or a THF solution of nonionic HPE at otherwise identical conditions, forming black non-redispersible precipitates. On the contrary, when dropped into the ionomer solution at a sufficiently high concentration [typically $\geq 0.1 \mathrm{mg} / \mathrm{mL}$ with $\left(m_{\text {ionomer }} / m_{\mathrm{Au}}\right)_{0} \geq 0.9$ ], no aggregation occurs and the GNRs remain well dispersed within the mixture. We reason that immediate destabilization/desorption of the CTAB bilayer occurs upon the addition of CTAB-coated GNRs into THF due to the enhanced critical micelle concentration of CTAB in the organic solvent. ${ }^{22}$ This is supported by the spontaneous GNR aggregation observed in the absence of the ionomer. In the presence of the ionomer, the simultaneous binding of the ionomer onto the GNRs occurs by displacing the surface-bound CTAB ligands, rendering stabilized ionomer-modified GNRs. Subsequently, the resulting ionomer-modified GNRs are subjected to multiple rounds (as high as 12 rounds herein; minimum 6) of centrifugation (at 11,176 g; $20 \mathrm{~min}$ ) and resuspension in fresh solvent (twice with ethanol/THF mixture then pure THF) to wash off CTAB and excess unbound or loosely bound ionomer. It has been known that alcohols can destabilize the CTAB bilayer,22,62 the wash of the ionomer-modified GNRs with THF/ethanol mixture is thus employed particularly to remove $\mathrm{CTAB}$ and render $\mathrm{CTAB}-$ free modified/functionalized GNRs. Meanwhile, the ionomer-modified GNRs disperse well in THF, which facilitates their wash with fresh THF for the removal of excess unbound or loosely bound ionomer. Scheme 2 shows schematically the modified GNRs.

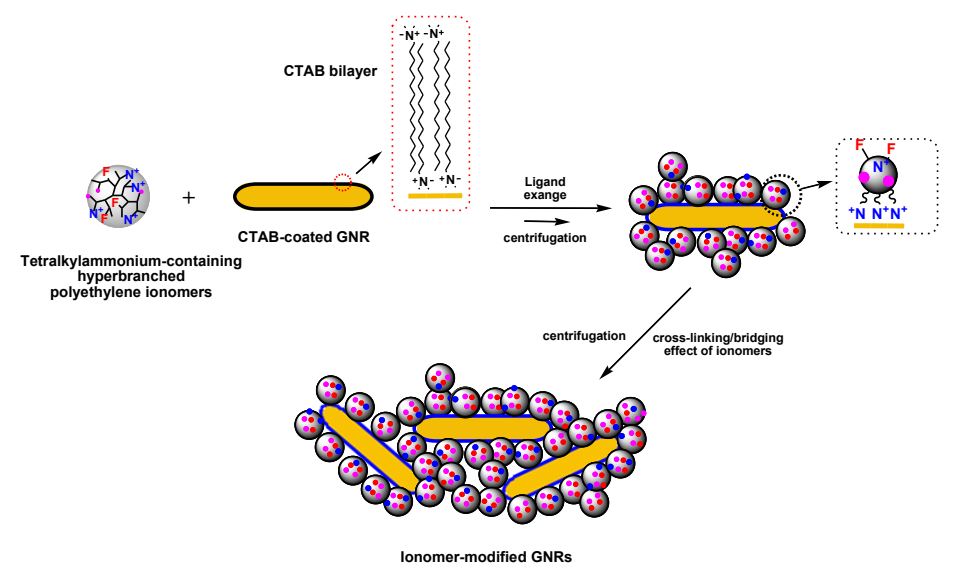

Scheme 2. Schematic surface modification of GNRs with hyperbranched polyethylene ionomers. 
With this direct dropping method, a range of ionomer-modified GNRs (I-GNR1 to I-GNR5 with I standing for ionomer modification and the number being consistent with the ionomer used) has been prepared from CTAB-coated short GNRs with the use of different pyrene-labeled ionomers (HPEI1-HPEI5, respectively). In particular, among them, I-GNR3-1 to I-GNR3-4 were prepared with HPEI3 at four different $\left(m_{\text {ionomer }} / m_{\text {Au }}\right)_{0}$ feed ratios $(0.2-1.7)$ to investigate the effect of the ratio on the ionomer modification. See Table 2 for other details on the preparation of these ionomer-modified GNRs.

Table 2. Ionomer-modified GNRs obtained with the use of different hyperbranched polyethylene ionomers.

\begin{tabular}{|c|c|c|c|c|c|c|c|}
\hline \multirow[b]{2}{*}{$\begin{array}{c}\text { Ionomer- } \\
\text { modified } \\
\text { GNRs }\end{array}$} & \multirow[b]{2}{*}{$\begin{array}{l}\text { CTAB- } \\
\text { coated } \\
\text { GNRs }\end{array}$} & \multirow[b]{2}{*}{ Ionomer } & \multirow[b]{2}{*}{$\begin{array}{c}{[\text { Ionomer }]_{0}^{a}} \\
(\mathrm{mg} / \mathrm{mL})\end{array}$} & \multirow[b]{2}{*}{$\begin{array}{c}\left(m_{\text {ionomer }} / m_{\mathrm{Au}}\right)_{0}{ }^{b} \\
(\mathrm{mg} / \mathrm{mg})\end{array}$} & \multicolumn{3}{|c|}{ Composition of ionomer-modified GNRs } \\
\hline & & & & & $\begin{array}{c}m_{\text {ionomer }} / m_{\mathrm{Au}}{ }^{c} \\
(\mathrm{mg} / \mathrm{mg})\end{array}$ & $\begin{array}{c}\text { Ionomer } \\
\text { mass } \\
\text { content }^{d}\end{array}$ & $\begin{array}{c}\text { Percentage of } \\
\text { ionomer } \\
\text { adsorption }^{e}\end{array}$ \\
\hline I-GNR1 & short & HPEI1 & 0.1 & 0.9 & 0.20 & 0.17 & 23 \\
\hline I-GNR2 & short & HPEI2 & 0.4 & 3.5 & 0.45 & 0.31 & 13 \\
\hline I-GNR3-1 & short & HPEI3 & 0.025 & 0.2 & 0.05 & 0.05 & 24 \\
\hline I-GNR3-2 & short & HPEI3 & 0.05 & 0.4 & 0.14 & 0.12 & 32 \\
\hline I-GNR3-3 & short & HPEI3 & 0.1 & 0.9 & 0.17 & 0.15 & 20 \\
\hline I-GNR3-4 & short & HPEI3 & 0.2 & 1.7 & 0.17 & 0.15 & 9 \\
\hline I-GNR4 & short & HPEI4 & 0.1 & 0.9 & 0.08 & 0.07 & 9 \\
\hline I-GNR5 & short & HPEI5 & 0.1 & 0.9 & 0.09 & 0.08 & 10 \\
\hline I-GNR6 & long & HPEI6 & 1 & 3.8 & 0.13 & 0.12 & 3.4 \\
\hline
\end{tabular}

${ }^{a}$ The feed concentration of ionomer solution in THF. ${ }^{b}$ The feed mass ratio of the ionomer to $\mathrm{Au} .{ }^{c}$ The mass ratio of the ionomer to Au in the resulting ionomer-modified GNRs determined from the fluorescence spectra of the modified GNRs. ${ }^{d}$ The mass fraction of the ionomer in the ionomer-modified GNRs. ${ }^{e}$ The percentage of the ionomer adsorbed onto the GNRs relative to its feed mass.

Designed on purpose with covalently tethered fluorescent pyrene groups, the ionomers impart the resulting modified GNRs with fluorescent properties, which in turn facilitates the convenient quantification of their content in the modified GNRs through fluorescence spectroscopy. Figure S5 in ESI shows the fluorescence (excitation and emission) spectra of representative ionomer- 
modified GNRs and their corresponding free ionomers in THF, as well as those of the pyrenecontaining monomer PMA. They all show the same characteristic fluorescent signals arising from the pyrene group, with the peak maxima of emission and excitation spectra at 374.5 and $341.5 \mathrm{~nm}$, respectively. In contrast, CTAB-coated GNRs do not show any fluorescent signals within the detection limits at identical conditions. With their fluorescent spectra, the concentration of the pyrene group and consequently the ionomer concentration in the dispersion of ionomer-modified nanorods are quantified by referring to calibration curves generated with PMA as the concentration standard (see Figure S6 in ESI). Along with the concentration of Au in the solution determined by atomic absorption spectroscopy, these data enable the determination of the mass content of the ionomer in the modified GNRs. In Table 2, the ionomer mass fraction and the ionomer/Au mass ratio $\left(m_{\text {ionomer }} / m_{\mathrm{Au}}\right)$ in the modified GNRs are summarized.

It has been known that fluorescence excitation and emission can be significantly altered when a fluorophore is near the plasmonic GNRs. ${ }^{68}$ In our system, such plasmonic effects on the fluorescence spectra of the ionomer-modified GNRs are ignored since their LSPR and TSPR bands are too far away from the fluorescence excitation/emission wavelengths to exert significant effects. To confirm this, we have also determined the amount of adsorbed ionomer indirectly by subtracting the ionomer feed amount with the amount of unadsorbed ionomer washed off during the purification procedure. In doing so, supernatant solutions collected during the purification procedure were monitored and quantified for unadsorbed ionomer with the fluorescence spectroscopy. In general, the amount of unadsorbed ionomer in the supernatants decreased quickly during the first three rounds of centrifugation, with no ionomers detected in supernatants obtained in subsequent rounds of centrifugation (see Table S2 in ESI). The ionomer content data obtained by both direct and indirect quantification methods are very close. For example, the direct method gives the ionomer mass fraction of 0.15 in I-GNR3-4 while the indirect method renders 0.17. In addition, we have also performed a thermogravimetric measurement on I-GNR2 (see Figure S7 in ESI). It shows a weight loss of $30.5 \mathrm{wt} \%$ within the temperature range of $250-500{ }^{\circ} \mathrm{C}$, which corresponds to the ionomer weight percentage. The value agrees well with the ionomer mass fraction of 0.31 found from the direct fluorescence measurement. 
Representatively, Figure 3a shows the UV-vis spectra (in THF) of HPEI3-modified GNRs, IGNR3-3, monitored after different rounds (4, 8, and 12, respectively) of centrifugation and resuspension during its purification/washing procedure, along with that of CTAB-coated short GNRs in aqueous phase. Despite the different rounds of centrifugation, the UV-vis spectra of the GNRs are almost identical with overlapping $\operatorname{LSPR}\left(\lambda_{\mathrm{LSPR}}=920 \mathrm{~nm}\right)$ and TSPR $\left(\lambda_{\text {TSPR }}=530 \mathrm{~nm}\right)$ bands, confirming the absence of irreversible nanorod aggregation under centrifugation. On the contrary, as-prepared CTAB-coated GNRs can generally sustain up to two rounds of centrifugation and resuspension in deionized water after their synthesis, with the occurrence of irreversible aggregation often in the third round of centrifugation. ${ }^{68,69}$ Previously, only few types of GNRs that are covalently functionalized with thiolated PEG and thiolated CTAB analogue can survive up to 5-6 rounds of centrifugation in water or in organic phase..$^{23,69}$ The high stability of the ionomer-modified GNRs herein is thus remarkable given that the nanorod stabilization is achieved exclusively through the noncovalent binding.
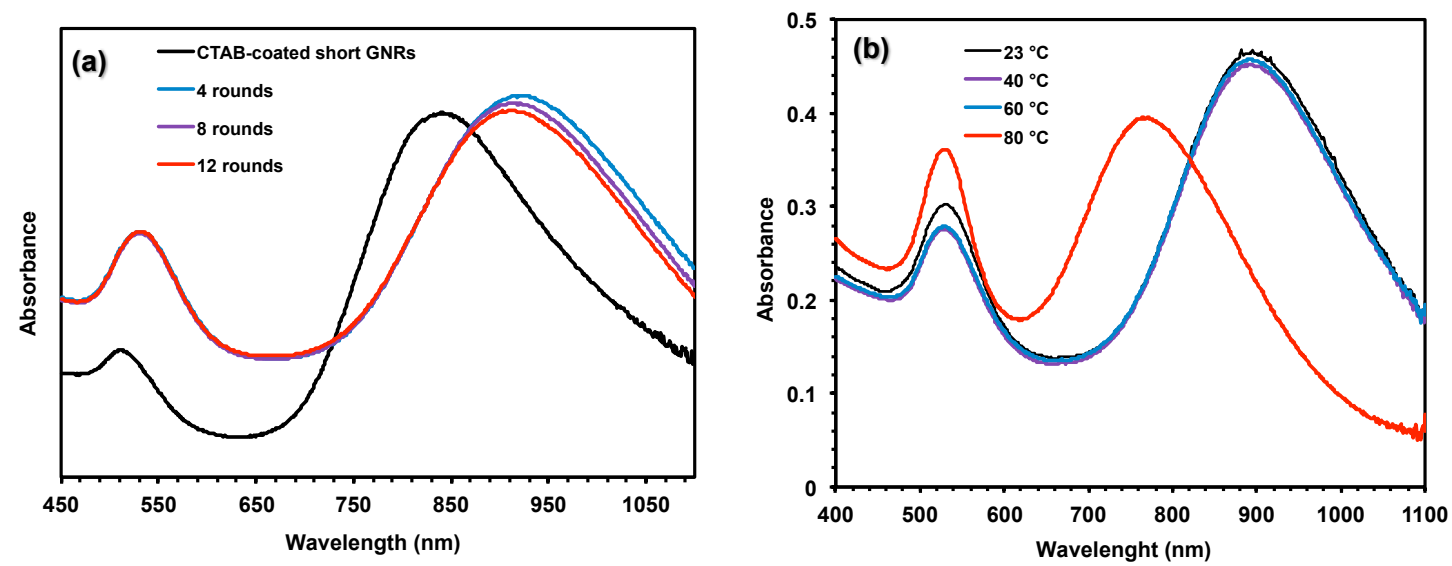

Figure 3. (a) UV-vis spectra (in THF after identical dilution) of HPEI3-modified short GNRs, IGNR3-3, after 4, 8, and 12 rounds, respectively, of centrifugation and resuspension monitored during its purification/washing procedure, along with the spectrum of CTAB-coated short GNRs in aqueous phase (after normalization with respect to its LSPR band) for comparison; (b) UV-vis spectra of I-GNR3-3 in THF at different temperatures.

We have further examined the stability of I-GNR3-3 (obtained after 12 rounds of centrifugation) in fresh THF at different temperatures $\left(23,40,60\right.$, and $\left.80{ }^{\circ} \mathrm{C}\right)$. Figure $3 \mathrm{~b}$ compares the UV-vis spectra of the dispersions after being kept at the four different temperatures for $20 \mathrm{~h}$. Raising the 
temperature from 23 to $60{ }^{\circ} \mathrm{C}$ causes negligible changes in the spectra, with the dispersions remaining stable without precipitates. But a significant blue shift of the LSPR band to $770 \mathrm{~nm}$ occurs with the solution kept at $80{ }^{\circ} \mathrm{C}$ along with the observation of some non-redispersible precipitates, indicating the occurrence of irreversible nanorod aggregation as well as possible particle shape change at this elevated temperature. Like the CTAB bilayer in CTAB-coated GNRs, we envisage that the multidentate binding of the ionomer to the GNR surface should also be dynamic, involving reversible association and dissociation. Herein, the major driving force for dissociation should be attributed to the ionomer-solvent interactions. Increasing the temperature likely enhances the ionomer-solvent interactions and meanwhile weakens the nonionic binding, leading to the possible desorption of some ionomers and thus nanorod aggregation. While no other GNRs have been previously investigated for their temperature stability, our results here confirm the well-retained stability of the ionomer-modified GNRs up to around $60{ }^{\circ} \mathrm{C}$.

Compared to CTAB-coated short GNRs $\left(\lambda_{\mathrm{LSPR}}=840 \mathrm{~nm}\right.$ and $\left.\lambda_{\mathrm{TSPR}}=510 \mathrm{~nm}\right)$, both of the LSPR and TSPR bands of I-GNR3-3 in Figure 3 show significant red shifts. Meanwhile, the relative intensity of the LSPR band (in reference to the TSPR band) is reduced after the ionomer coating. This trend of change is also observed with ionomer-modified GNRs prepared from other ionomers (see Figure 4a). While the increase in local refractive index should lead to the red shift of the LSPR band only and its enhanced intensity as shown in the previous section, the red shifts of both bands and the reduced relative intensity of the LSPR band herein suggest the presence of inter-nanorod plasmon coupling as a result of the reduced inter-nanorod distance, in addition to the effects resulting from the change in solvent refractive index. ${ }^{61}$ Though the precise mechanism is to be investigated through further study, we reason that this is attributed to the bridging effect of the multidentate ionomers (see Scheme 2). We envision that different quaternary ammonium groups on the same ionomer chain may bind to different GNRs when the GNR concentration is high; meanwhile, those unbound ammonium groups on ionomer chains adsorbed to different GNRs may also join together to form ionic aggregates (see Scheme 2). The ionomers can thus act as macromolecular "cross-linkers" and bring the GNRs to a closer distance during high-speed centrifugation, while without causing irreversible aggregations due to the presence of the ionomers in the interstices. The absence of inter-nanorod plasmon coupling with the GNRs 
following phase transfer (in Figure 2) is because they were not subjected to high-speed centrifugation.

Following the above reasoning, the plasmon coupling resulting from the bridging or crosslinking effect of the ionomers should be more pronounced with the increase of the content of the quaternary ammonium groups in the ionomer, due to their increased number of available sites for "cross-linking". This is corroborated by the UV-vis spectrum of I-GNR5 (in Figure 4a) prepared with HPEI5 having the highest quaternary ammonium content $(1.8 \mathrm{~mol} \%)$. In addition to the red shifts of both bands, I-GNR5 shows greatly broadened LSPR and TSPR bands, along with the formation of non-redispersible precipitates during its purification procedure. On the contrary, GNRs modified with other ionomers do not show obvious band broadening though with red shifts. On the basis of these results, ionomers with quaternary ammonium content $\leq 1.0 \mathrm{~mol} \%$ (i.e., HPEI1-HPEI4) in this set should be used in order to obtain stable modified GNRs.
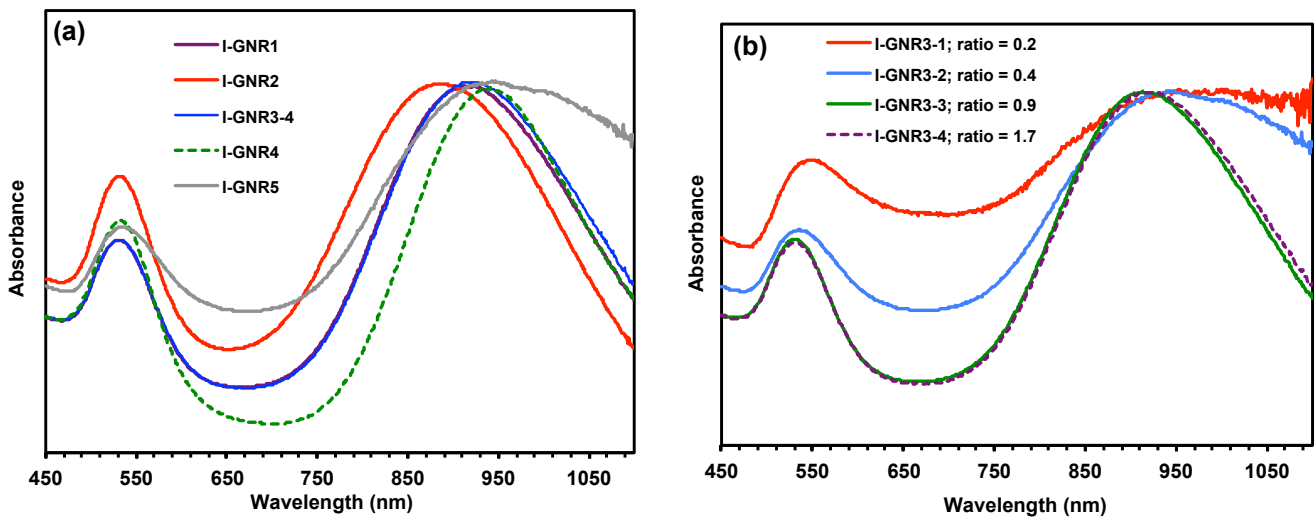

Figure 4. (a) UV-vis spectra of various ionomer-modified short GNRs (I-GNR1 to I-GNR5 in THF) prepared from CTAB-coated short GNRs with different HPEIs; (b) UV-vis spectra of HPEI3-modified GNRs (I-GNR3-1 to I-GNR3-4 in THF) prepared with HPEI3 at different $\left(m_{\text {ionomer }} / m_{\mathrm{Au}}\right)_{0}$ feed ratios. The spectra are normalized with respect to their LSPR bands.

The $\left(m_{\text {ionomer }} / m_{\mathrm{Au}}\right)_{0}$ feed ratio employed in the direct dropping method has significant effect on the ionomer coating. Figure 4b compares the UV-vis spectra of I-GNR3-1 to I-GNR3-4 prepared with HPEI3 at different feed ratios (0.2 to 1.7). For I-GNR3-1 and I-GNR3-2 prepared at lower feed ratios ( 0.2 and 0.4 , respectively), significant aggregation of the nanorods can be seen on the 
basis of the severe broadening in the LSPR and TSPR bands, as well as the observation of some black non-redispersible precipitates formed during their purification. In particular, between the two, the band broadening is even worse in I-GNR3-1 prepared at the lowest feed ratio. However, for I-GNR3-3 and I-GNR3-4 prepared at higher feed ratios (0.9 and 1.7, respectively), their UVvis spectra are nearly identical without obvious band broadening, meanwhile with no formation of non-redispersible precipitates. These results indicates the insufficient ionomer coating in IGNR3-1 and I-GNR3-2 prepared at $\left(m_{\text {ionomer }} / m_{\mathrm{Au}}\right)_{0}<0.9$. By comparing the UV-vis spectra of IGNR3-3 and I-GNR3-4, it appears that the feed ratio has no effect on the ionomer coating once above 0.9. Quantitative analysis on the ionomer mass content in the modified GNRs to be shown below also confirms this. Sufficient ionomer feed with $\left(m_{\text {ionomer }} / m_{\mathrm{Au}}\right)_{0} \geq c a .0 .9$ is thus necessary in this direct dropping method in order to warrant the stability of the resulting ionomer-modified GNRs.

To investigate if CTAB is still present on the modified GNRs prepared through this method, we have used ${ }^{1} \mathrm{H}$ NMR spectroscopy to analyze the organic surface ligands present in I-GNR2 as a representative modified GNR sample. We initially attempted to perform the ${ }^{1} \mathrm{H}$ NMR characterization on the ionomer-modified GNRs directly. However, no signals were captured except those of the solvents despite several trials, possibly due to the effects of the metal nanoparticles. An indirect method was then employed. The gold core in I-GNR2 was dissolved with $\mathrm{NaCN}$ to release the surface-bound organic material into the organic solution (methanol/ $\mathrm{CDCl}_{3}$ mixture) for characterization. Figure 5 shows the ${ }^{1} \mathrm{H} \mathrm{NMR}$ spectrum, along with that of pure CTAB in the same solvent mixture. Strong signals attributable to the hyperbranched polyethylene skeletons $(0.75,1.14$, and $1.18 \mathrm{ppm}$ for methyl, methine, and methylene protons, respectively) ${ }^{45,46,48-55}$ are present in the spectrum of I-GNR2. Signals characteristic of CTAB are absent within the detection limit of ${ }^{1} \mathrm{H}$ NMR spectroscopy. This spectroscopic evidence thus suggests the quantitative displacement of surface-bound CTAB by the ionomers. ${ }^{23}$ The direct dropping method with purification through multiple rounds of centrifugation is thus effective in rendering CTAB-free ionomer-modified GNRs. Previously, quantitative CTAB replacement has only been reported with GNRs covalently modified with a thiolated CTAB analogue ${ }^{23}$ while GNRs prepared by other methods all contain residue CTAB. ${ }^{6}$ 


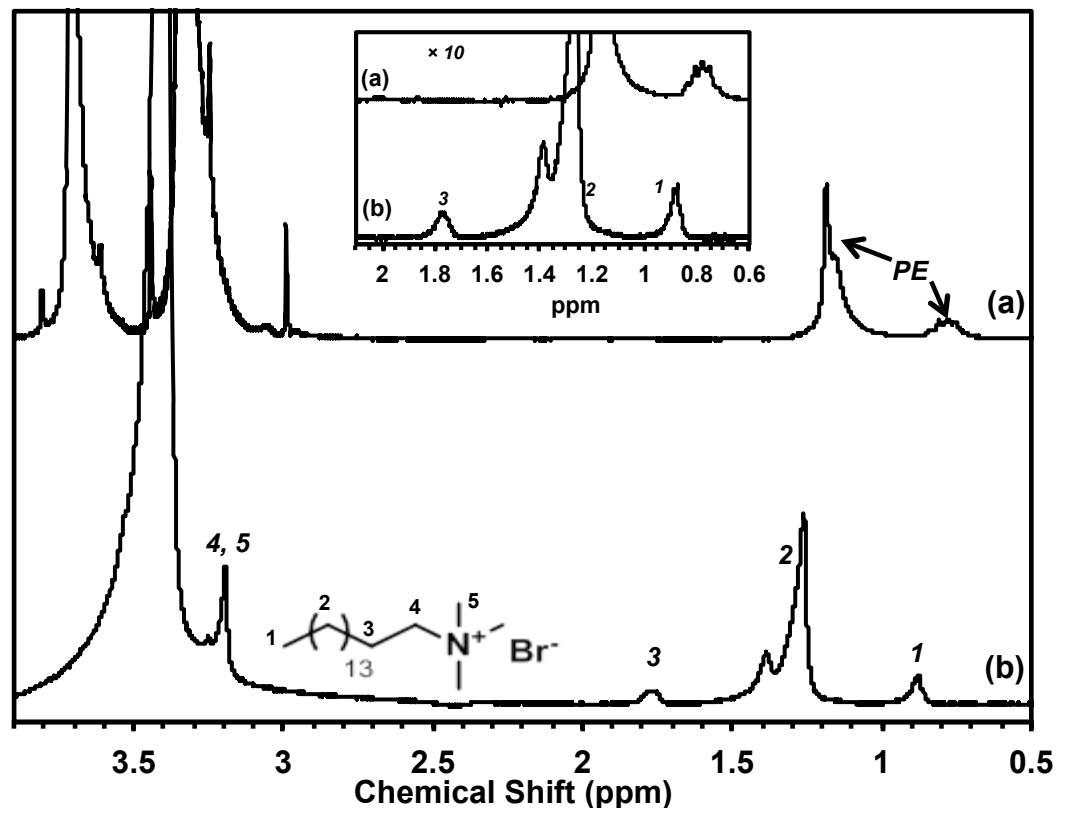

Figure 5. ${ }^{1} \mathrm{H}$ NMR spectrum of the organic surface ligands in I-GNR2 after digestion of gold core (a), along with that of pure CTAB (b). The NMR characterization was performed with mixed $\mathrm{MeOH} / \mathrm{CDCl}_{3}$ as solvent.

Figure 6 shows the TEM images of various ionomer-modified GNRs, which were taken on the specimen laid on the carbon film of the lacey TEM grids. On the basis of these images, the nanorod morphology has been well retained after ionomer modification without disruptive aggregation. Though some spherical particles are also seen, they should be those formed during the large-scale seed-mediated GNR synthesis instead of being formed during the ionomer modification procedure. Due to the presence of the thick underlying carbon film, the presence of ionomers in the composites could not be visualized in these images due to their low density and amorphous nature. In consequence, images have also been taken on areas with specimen hanging out into grid openings without the underlying carbon film. Figure 7 shows such images of the representative ionomer-modified GNRs, where the presence of ionomers can be clearly visualized. From these images, large composite lumps can be seen, with the nanorods interspersed within the ionomer. The ionomer mass content (up to $31 \mathrm{wt} \%$ ) is significant in the modified GNRs while with much lower density compared to the GNRs, leading to the high volumetric content of the ionomers. These composite lumps should be formed by conglomeration of the specimen upon solvent evaporation during the TEM sample preparation 
since the ionomers are highly sticky with low glass transition temperature $\left(-65^{\circ} \mathrm{C}\right)$ in their solid state. $^{55}$
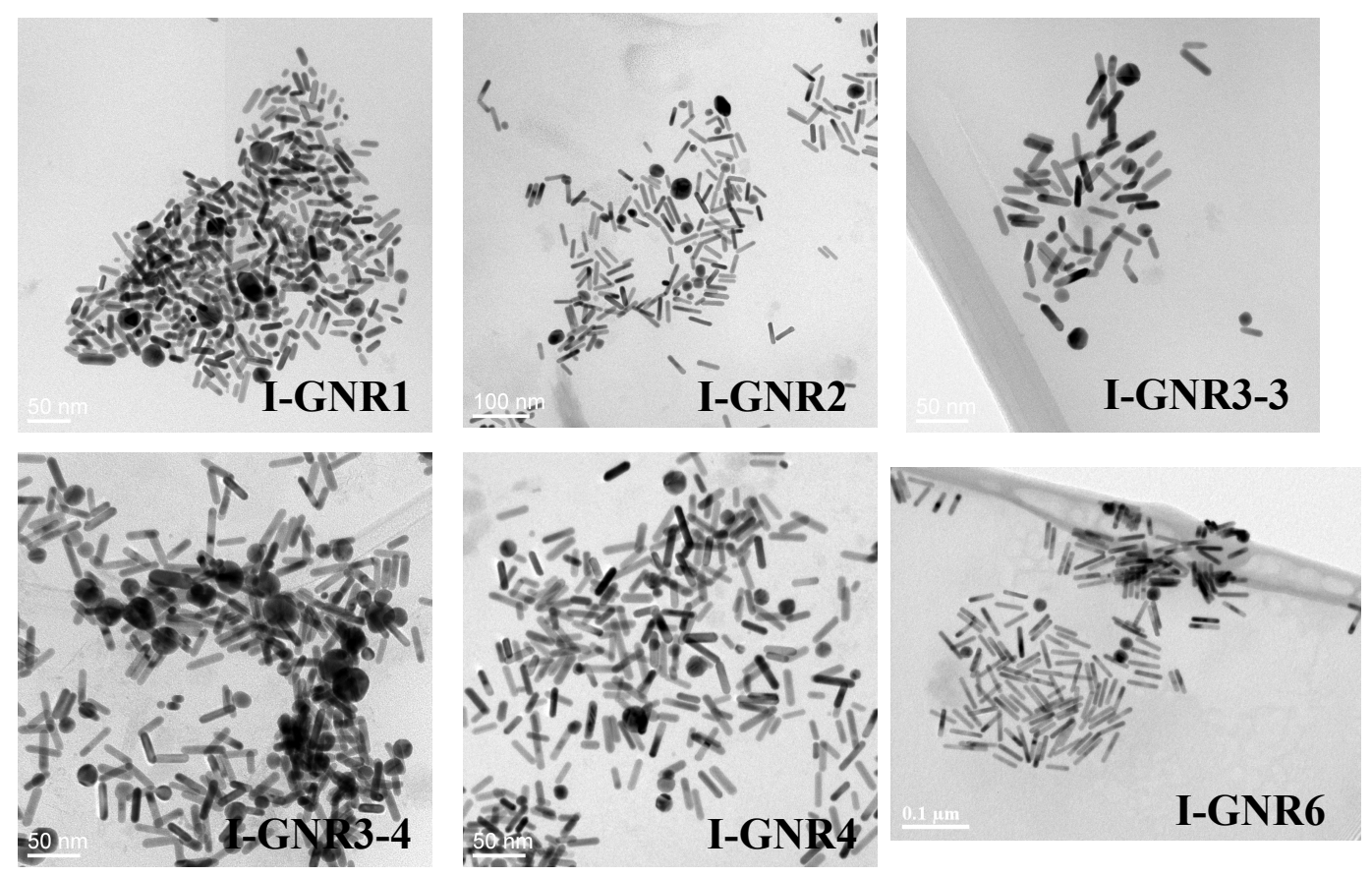

Figure 6. TEM images of the various ionomer-modified GNRs, I-GNR1 to I-GNR4 prepared from CTAB-coated short GNRs and I-GNR6 prepared from long GNRs. The images were taken from the specimen laid on the carbon film of the TEM grids.
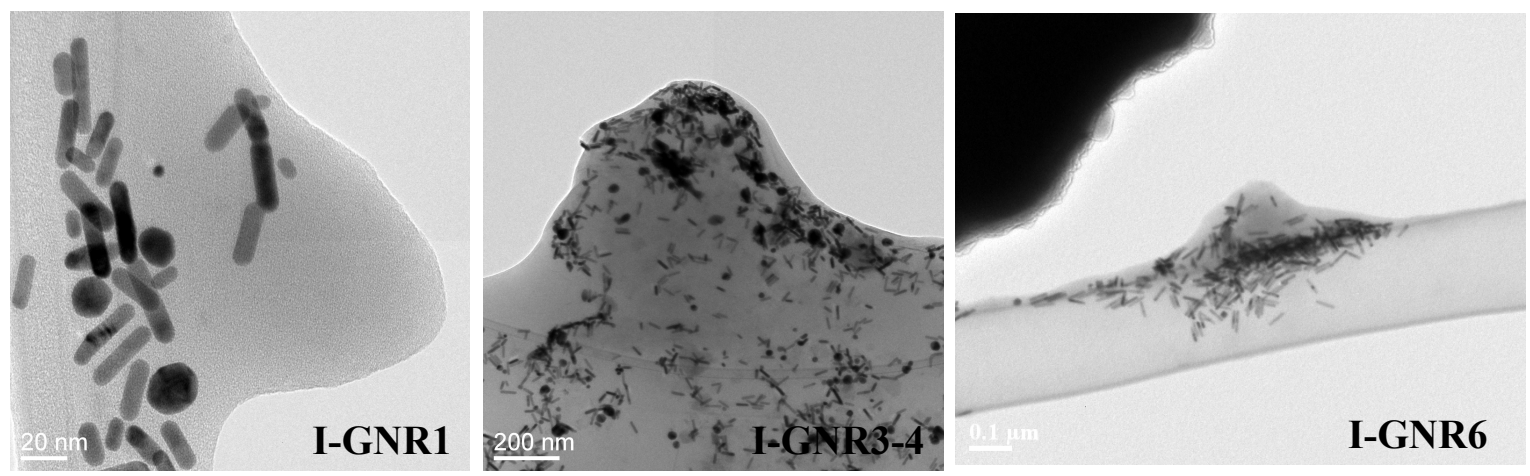

Figure 7. TEM images for representative ionomer-modified GNRs, showing the presence of the ionomers. The images were taken on the specimen hanging out into grid holes without the interference of the carbon film. 
We then discuss the effects of the $\left(m_{\text {ionomer }} / m_{\mathrm{Au}}\right)_{0}$ feed ratio and different ionomers on the ionomer adsorption. Figure 8a plots the dependence of the $m_{\text {ionomer }} / m_{\text {Au }}$ ratio in the modified GNRs on $\left(m_{\text {ionomer }} / m_{\mathrm{Au}}\right)_{0}$ ratio for I-GNR3-1 to I-GNR3-4 prepared with the same ionomer, HPEI3. A gradual increase in the $m_{\text {ionomer }} / m_{\text {Au }}$ ratio to a plateau value of 0.17 is noted with the increase of the $\left(m_{\text {ionomer }} / m_{\mathrm{Au}}\right)_{0}$ ratio to 0.9 ; after that, the ionomer content remain unchanged despite the further increase of ionomer feed. This trend of change indicates the presence of a maximum ionomer binding on the nanorod surface, at which the nanorod surface is fully covered and the further binding of additional ionomer is not possible. This is consistent with the results from their UV-vis spectra (see Figure $4 \mathrm{~b}$ and associated discussion above).
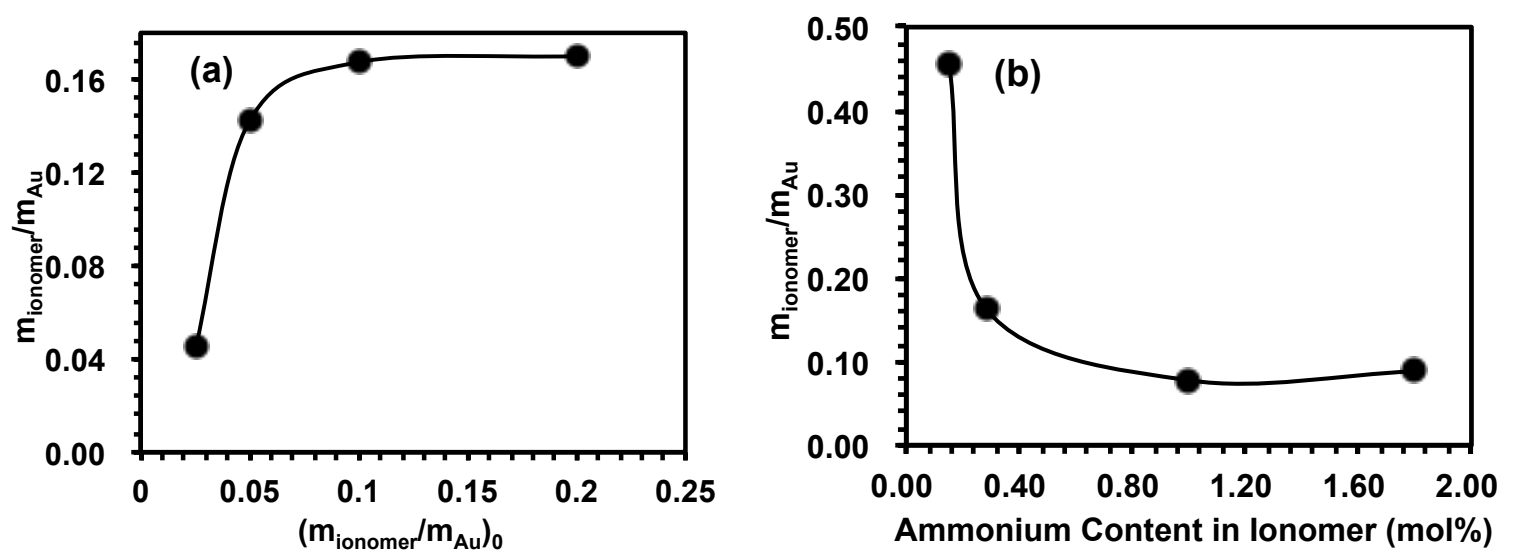

Figure 8. (a) Effect of ionomer/Au feed ratio, $\left(m_{\text {ionomer }} / m_{\mathrm{Au}}\right)_{0}$, on the $m_{\text {ionomer }} / m_{\mathrm{Au}}$ ratio in the HPEI3-modified GNRs (I-GNR3-1 to I-GNR3-4); (b) effect of ionomers with different quaternary ammonium content (HPEI2-HPEI5) on the $m_{\text {ionomer }} / m_{\mathrm{Au}}$ ratio in the resulting ionomermodified GNRs (I-GNR2, I-GNR3-4, I-GNR4, and I-GNR5).

Figure $8 \mathrm{~b}$ shows the effect of different ionomers (HPEI2-HPEI5) with varying quaternary ammonium content on the $m_{\text {ionomer }} / m_{\mathrm{Au}}$ ratio in the resulting modified GNRs (i.e., I-GNR2, IGNR3-4, I-GNR4, and I-GNR5). Prepared at high $\left(m_{\text {ionomer }} / m_{\mathrm{Au}}\right)_{0}$ ratios $(\geq 0.9)$, these modified GNRs should have reached their respective maximum ionomer adsorption. A drastic decrease in the $m_{\text {ionomer }} / m_{\mathrm{Au}}$ ratio from 0.45 to 0.08 (i.e., decreasing ionomer mass fraction) can be noted with the increase of ammonium content from 0.15 (HPEI2) to $1.0 \mathrm{~mol} \%$ (HPEI4). This suggests that, at a given mass fraction, ionomers of a higher ammonium content have more binding sites for 
adsorption onto rod surface. The further increase of the ammonium content to $1.8 \mathrm{~mol} \%$ (HPEI5), however, does not cause a noticeable change in the $m_{\text {ionomer }} / m_{\mathrm{Au}}$ ratio. Consistent with the UV-vis spectrum of I-GNR5 shown in Figure 4a, the use of ionomers of high quaternary ammonium ion content (like HPEI5) is detrimental to the stability of the resulting modified GNRs because of the insufficient ionomer binding on the GNR surface to prevent their aggregation during their purification by centrifugation.

Besides THF where the modified GNRs can dissolve well, we have further examined the dispersibility of the modified GNRs in a broad range of other organic solvents. Due to the presence of the ionomer modification layer, the modified GNRs have been found to be dispersible in several solvents of varying polarity. Figure 9 shows the photographs of the stable dispersions of I-GNR3-3 as a representative sample in different solvents and their UV-vis spectra. Not only dispersible in nonpolar or low-polarity solvents (including toluene, chlorobenzene, THF and chloroform where the pure ionomer can dissolve well) as already shown above, the modified GNRs are also dispersible in other polar solvents including dioxane, acetonitrile, and N-methyl-2-pyrrolidone (NMP), where the pure ionomer does not show good solubility. The various dispersions were found stable for at least 3 months without the formation of irreversible precipitates. Their UV-vis spectra show the distinct LSPR and TSPR bands but with red shifts relative to those of CTAB-coated short GNRs in water. While their TSPR bands all locate at $\lambda_{\text {TSPR }}=c a .530 \mathrm{~nm}$, their LSPR bands have different $\lambda_{\text {LSPR }}$ depending sensitively on the solvent, but generally with maintained shape and minimum broadening. From the inset in Figure 9, their $\lambda_{\text {LSPR }}$ shows a trend of increase with the increase of solvent refractive index, with the highest $\lambda_{\text {LSPR }}$ of $925 \mathrm{~nm}$ found in chlorobenzene. Previously, polyelectrolyte-coated GNRs have been shown to be dispersible in a range of highly polar organic solvents. ${ }^{32}$ However, it has not been shown if they can survive multiple rounds of centrifugation in those solvents. Meanwhile, no other modified GNRs have the demonstrated solubility in the various non-polar or low-polarity solvents presented herein.

When dispersed in some other organic solvents (including hexane, dimethylformamide (DMF), dichloromethane, and ethyl acetate), irreversible aggregation was, however, found to occur with the formation of non-redispersible large precipitates after standing overnight. Figure S8 in ESI 
shows the UV-vis spectra of I-GNR3-3 taken shortly after the dispersion in these solvents. Significant broadening of both LSPR and TSPR bands with changes in their shapes can be noticed, along with the observation of non-redispersible precipitates, indicating the presence of severe nanorod aggregation. The nanorod aggregation found in these solvents may result from either the possible desorption of ionomers due to too strong solvent-ionomer interactions (in the case hexane and ethyl acetate) or the insufficient solvent-ionomer interactions which are required to maintain nanorod stability (in the case of DMF and dichloromethane).

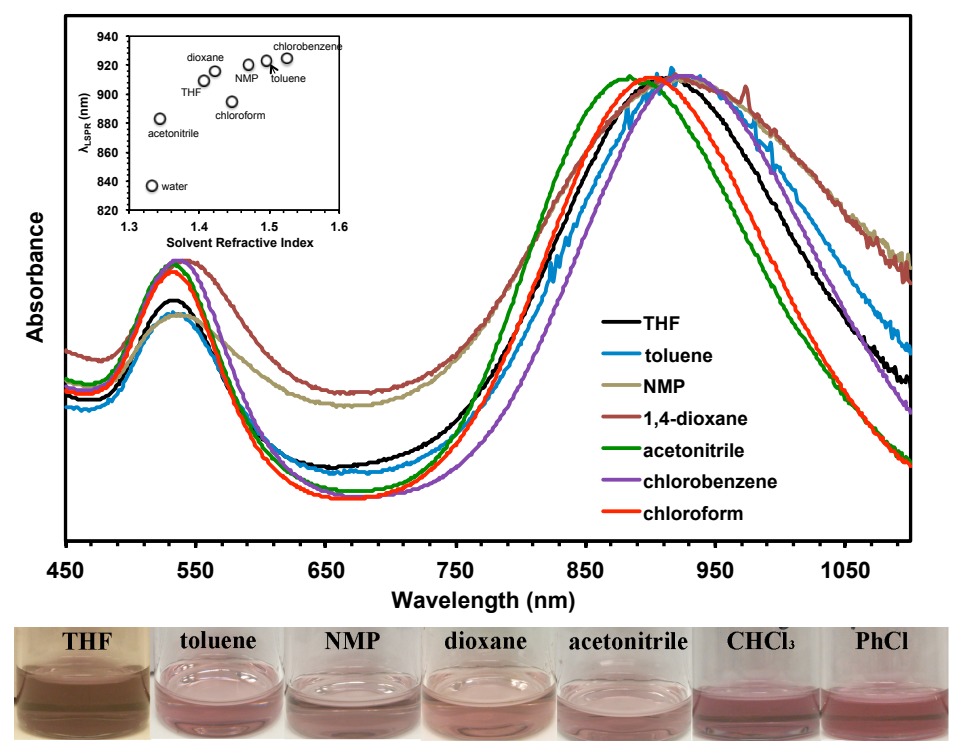

Figure 9. UV-vis spectra and images of I-GNR3-3 dispersed in different organic solvents. The dispersions $(0.023 \mathrm{mg} \mathrm{Au} / \mathrm{mL})$ were prepared by adding a small volume of THF solution of IGNR3-3 into the corresponding solvent (1:9 volume ratio), followed with one round of centrifugation $(11,176 \mathrm{~g} ; 20 \mathrm{~min})$ and resuspension in the corresponding pure solvent. The spectra are normalized with respect to their LSPR bands.

Preparation of Amphiphilic Modified GNRs by Direct Dropping Method and Their Use as Carrier of Hydrophobic Guest Species

Though dispersible in the above organic solvents, the modified GNRs prepared with HPEI1HPEI5 are not dispersible in water or some other high-polarity solvents (such as ethanol, acetone, etc.) due to the hydrophobic nature of the hyperbranched polyethylene skeleton and the absence of sufficient hydrophilic segments in the ionomers. To further demonstrate the 
versatility of this ionomer modification strategy, we have subsequently extended to prepare amphiphilic ionomer-modified GNRs, which show dispersibility in both aqueous and various organic media, so as to broaden their possible applications in aqueous/biological media. For this purpose, we have particularly designed HPEI6, a tetrapolymer of ethylene, $\mathrm{AETA}^{+} \mathrm{BF}_{4}^{-}, \mathrm{PMA}$, and OEGA. Containing a significant fraction (11 mol\%, see Table 1 for its composition) of OEGA with a pendant amphiphilic OEG segment, HPEI6 is amphiphilic with good solubility in both water and various nonpolar, low-polarity, or high-polarity organic solvents (such as ethanol, acetone, toluene, THF, etc.). Meanwhile, it has an optimized high quaternary ammonium content of $1.7 \mathrm{~mol} \%$, which has been found necessary in order to render stable modified GNRs due to the high mass content of OEGA in the ionomer.

HPEI6-modified GNRs, I-GNR6, were similarly prepared from CTAB-coated long GNRs by the direct dropping method. Through fluorescence spectroscopy, the resulting modified GNRs were found to have a $m_{\text {ionomer }} / m_{\mathrm{Au}}$ ratios of 0.13 (i.e., ionomer mass fraction of 0.12 ). Given the high $\left(m_{\text {ionomer }} / m_{\mathrm{Au}}\right)_{0}$ feed ratio, the maximum HPEI6 adsorption should be achieved in the modified GNRs. Representative TEM images of I-GNR6 are also included in Figures 6 and 7, confirming the integrity of the nanorod morphology after the modification and the presence of the ionomer in the composite.

Figure 10 shows the UV-vis spectra and photographs of the dispersions of I-GNR6 in water and ten organic solvents, including nonpolar or low-polarity ones (toluene, chlorobenzene, ethyl acetate, THF, chloroform, dioxane) and high-polarity ones (ethanol, acetone, DMF, and Nmethyl-2-pyrrolidone (NMP)). The dispersions were all stable for at least 3 months without the formation of non-redispersible precipitates. Their UV-vis spectral shapes are similar to that of CTAB-coated long GNRs in water. Their TSPR bands all locate at $\lambda_{\text {TSPR }}=c a .510 \mathrm{~nm}$ with negligible shifts relative to that of original CTAB-coated GNRs; their LSPR bands show shifts with minimum broadening (except in DMF with significant broadening) within the recorded wavelength range, which corresponds to the change in refractive index of the solvents. The inset in Figure 10(a) confirms that their $\lambda_{\text {LSPR }}$ increases with the increase of the solvent refractive index. It is interesting to note that the LSPR band of I-GNR6 in water shows a blue shift $\left(\lambda_{\text {LSPR }}=\right.$ $898 \mathrm{~nm}$ vs. $983 \mathrm{~nm}$ ) relative to that of original CTAB-coated GNRs in water. With the same 
solvent used, this blue shift reflects the difference in their surface ligands. In the high-polarity solvents, we reason that the hyperbranched polyethylene segments in the ionomer are collapsed on the nanorod surface due to their highly hydrophobic nature, while with the hydrophilic OEG segments protruding out into the solvent to render the stability of the modified nanorods. The spectral evidence confirms the amphiphilic nature of I-GNR6 and its stable dispersion in both water and a broad range of organic solvents with varying polarity.
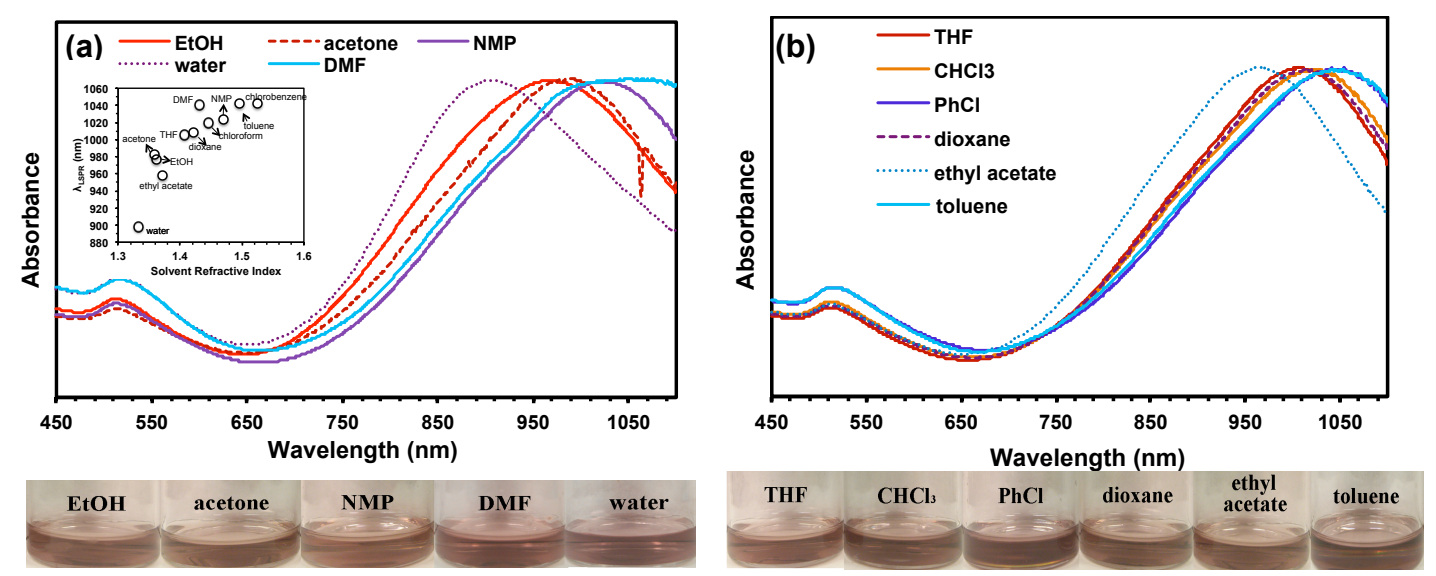

Figure 10. UV-vis spectra and photographs of I-GNR6 dispersions (containing $0.02 \mathrm{mg} \mathrm{Au} / \mathrm{mL}$ ) in water and various polar solvents (a) and low-polarity or nonpolar solvents (b). The spectra are normalized with respect to the LSPR bands. The dispersions were prepared by adding a small volume of ethanol solution of I-GNR6 into the corresponding solvent (1:9 volume ratio), followed with one round of centrifugation $(11,176 \mathrm{~g}$ for $20 \mathrm{~min})$ and resuspension in the corresponding pure solvent.

The aqueous dispersion of I-GNR6 was characterized with dynamic light scattering (DLS) for the particle size and $\zeta$-potential. The CTAB-coated long GNRs show an average hydrodynamic size of $c a .105 \mathrm{~nm}$. I-GNR6 instead exhibits a significantly larger average size of $c a .370 \mathrm{~nm}$. Relative to the value of +36 for the CTAB-coated long GNRs, I-GNR6 has a reduced $\zeta$-potential of +19 , reflective of the reduced charge upon the modification with the ionomer having a lower ionic content compared to CTAB. These DLS data also provide the evidence of the ionomers adsorbed on the nanorod surface, which changes their particle size and $\zeta$-potential. 
Containing both hydrophobic and hydrophilic chain segments, both the amphiphilic ionomer HPEI6 and the modified GNRs as HPEI6/GNR composites, when dispersed in water, are reasoned to form self-assembled structures containing hydrophobic polyethylene domains surrounded by a hydrophilic OEG shell. ${ }^{70}$ We hypothesized that both the ionomer and the modified GNRs dispersed in water should be able to act as nanoscale carrier for hydrophobic guest species by their encapsulation within the hydrophobic polyethylene domains. As a proofof-concept, we have used Nile Red, a common hydrophobic dye and an excellent fluorescence probe, for its encapsulation within I-GNR6 and HPEI6 dispersed in water. Nile Red is insoluble and does not fluoresce in water; but once encapsulated, its aqueous solution starts to fluoresce. It has often been used to examine the encapsulation efficiency of different types of nanocarriers. ${ }^{70}$

Figure S9 in ESI shows the fluorescence spectra of the water dispersions of HPEI6 (concentration: $0.5 \mathrm{mg} / \mathrm{mL}$ ) and I-GNR6 (concentration: $5.7 \mathrm{mg} / \mathrm{mL}$ with adsorbed HPEI6 at $0.68 \mathrm{mg} / \mathrm{mL}$ ) before and after the addition of excess Nile Red for encapsulation. While the dispersions before Nile Red addition do not fluoresce, strong characteristic fluorescence signals arising from Nile Red can be found with dispersions following Nile Red addition, thus confirming the encapsulation of Nile Red within both HPEI6 and I-GNR6. Quantification of the fluorescence spectra by referring to a calibration curve (see Figure S10 in ESI) indicates that the HPEI6 and I-GNR6 dispersions contain encapsulated Nile Red at a concentration of 0.23 and $0.16 \mathrm{mg} / \mathrm{L}$, respectively, in the dispersions. Correspondingly, the Nile Red encapsulation capacity within HPEI6 and I-GNR6 is $0.046 \mathrm{wt} \%$ of mass of HPEI6 and $0.0028 \mathrm{wt} \%$ of mass of I-GNR6, respectively (i.e., $1.43 \mu \mathrm{mol} / \mathrm{g}$ of HPEI6 and $0.09 \mu \mathrm{mol} / \mathrm{g}$ of I-GNR6, respectively). In particular, relative to the mass of HPEI6 present in I-GNR6, the encapsulation capacity is 0.024 wt $\%$ or $0.8 \mu \mathrm{mol} / \mathrm{g}$, which are in the same order of the corresponding encapsulation values achieved with pure HPEI6. Though the encapsulation capacity is yet to be improved via further design, these preliminary results confirm the capability of the amphiphilic ionomer-modified GNRs as the carrier of hydrophobic guest species. Meanwhile, the finding also sheds light on the potential design of the amphiphilic ionomer-modified GNRs as unique multifunctional drug carriers with photothermal GNR cores and fluorescent labels for applications in cancer therapeutics. 


\section{Modification of GNRs with a Linear Poly(n-Butyl Acrylate) Ionomer}

In the above studies, we have used exclusively hyperbranched polyethylene-based ionomers (HPEI0-HPEI6). We have found that this ionomer functionalization strategy also works well with other quaternary ammonium-containing ionomers built with different polymer skeletons, such as PBAI with a linear poly( $n$-butyl acrylate) skeleton. PBAI-modified GNRs were prepared from CTAB-coated long GNRs via the direct dropping method at the $\left(m_{\mathrm{PBAI}} / m_{\mathrm{Au}}\right)_{0}$ feed ratio of 2.5 , followed with multiple rounds of centrifugation. As per the quantification by fluorescence spectroscopy, the resulting modified GNRs have an $m_{\mathrm{PBAI}} / m_{\mathrm{Au}}$ ratio of 0.24 or the PBAI mass fraction of 0.19. Figure 11 shows the UV-vis spectra of the modified GNRs dispersed in three representative organic solvents, THF, acetone, and ethanol. In all the three solvents, the modified GNRs are stably dispersed and the shape of their UV-vis spectra well resembles that of original CTAB-coated GNRs in water. Their TSPR bands are all located at $510 \mathrm{~nm}$ with no shifts compared to that of original CTAB-coated GNRs; the LSPR bands show red shifts depending on the solvent. We also note that their $\lambda_{\text {LSPR }}$ values are different from those of I-GNR6 dispersed in the same respective solvents (see Figure 11), indicating that different ionomers adsorbed on the GNR surface affects the local refractive index around the GNRs. There is very minor broadening with the LSPR bands of the modified GNRs in acetone and ethanol perhaps because PBAI itself does not dissolve well in the two solvents. After normalization with respect to the TSPR bands, their LSPR bands are all found to have similar intensity as that of original CTAB-coated GNRs. This suggests the negligible nanorod aggregation or plasmon coupling in the PBAI-modified GNRs. While plasmon coupling is present with I-GNR1 to I-GNR4, its absence herein is possibly related to the linear skeleton of the PBAI ionomer as opposed to the highly compact hyperbranched ones of HPEI1-HPEI4. At the same ionomer mass fraction, the linear skeleton of PBAI is reasoned to render the greater inter-rod distance due to its significantly less compact chain conformation and thus the disappearance of plasmon coupling. 


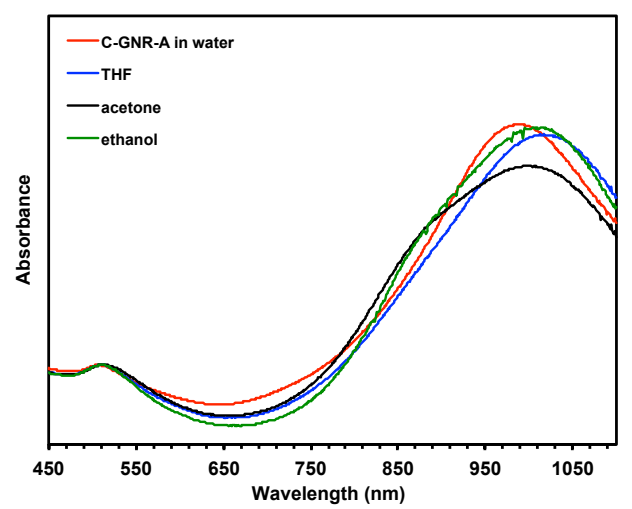

Figure 11. UV-vis spectra of PBAI-modified GNRs in different solvents, along with that of CTAB-coated long GNRs in water for comparison. The spectra are normalized with respect to their TSPR bands at $510 \mathrm{~nm}$.

\section{Conclusions}

We have demonstrated a new strategy for efficient and robust surface functionalization of GNRs with the use of quaternary ammonium-containing ionomers as multidentate macromolecular surface ligands. A range of ionomers, including hyperbranched polyethylene ionomers (HPEIs including HPEI0-HPEI6) and the linear poly(n-butyl acylate) ionomer (PBAI) containing tetralkylammonium ions along with other functional groups (fluorescent pyrene groups or amphiphilic OEG blocks), have been tailor-designed in this work to illustrate the efficiency of the strategy.

Acting as the macromolecular $\mathrm{CTAB}$ analogues with multiple binding sites, the ionomers have been found to bind strongly onto the nanorod surface by displacing surface-bound CTAB ligands through ligand exchange to form the ionomer modification layer. With HPEIO as the example, we have successfully shown the nearly complete phase transfer of the GNRs from the aqueous phase to multiple nonpolar or low-polarity organic phases, while without experiencing nanorod aggregation. A simple direct dropping method has been further developed to conveniently render various ionomer-modified GNRs (I-GNR1 to I-GNR6) with the use of corresponding ionomers (HPEI1-HPEI6). With the hydrophobic HPEI ionomers, the quaternary ammonium content should be designed to be $\leq 1.0 \mathrm{~mol} \%$ (i.e., HPEI1-HPEI4) in order to obtain stable modified 
GNRs. TEM images confirm the presence of the adsorbed ionomers in the modified GNRs. As per the ${ }^{1} \mathrm{H}$ NMR characterization of the surface ligands present in I-GNR2, the modified GNRs are CTAB-free with quantitative displacement of CTAB by the ionomers. The quantitative study on the adsorption of the ionomer on nanorod surface shows the dependencies on both the $\left(m_{\text {ionomer }} / m_{\mathrm{Au}}\right)_{0}$ feed ratio and the quaternary ammonium content in the ionomers. The ionomer with a lower quaternary ammonium content tends to render its higher mass content in the modified nanorods.

Because of the adsorbed ionomers, the modified GNRs (I-GNR1 to I-GNR4, and I-GNR6) have been found to be highly stable and survive multiple (up to 12 investigated herein) rounds of highspeed centrifugation in organic solvents while without incurring aggregation. The adsorbed ionomer also renders the modified GNRs the dispersibility in various organic solvents with different polarity. In particular, I-GNR6 modified with amphiphilic HPEI6 is also amphiphilic with excellent dispersibility in both aqueous and a broad range of organic solvent media. Through the experiment on its encapsulation of Nile Red, we have also shown that I-GNR6, when dispersed in water, can act as a carrier for hydrophobic guest species, which renders its potential as multifunctional GNR-embedded composite drug carrier in biological applications.

Demonstrated with HPEIs and PBAI as representative ionomers, this new strategy for GNR functionalization with quaternary ammonium-containing ionomers should be general. Meanwhile, this strategy should also be applicable for the functionalization of other metallic nanoparticles. With the availability of numerous monomers with valuable functionalities and enabling polymerization techniques, various desired quaternary ammonium-containing ionomers as a new class of macromolecular surface ligands can thus be tailor designed to suit specific multifunctional applications. This strategy thus offers a new toolbox of functional ionomers as multidentate macromolecular ligands for efficient, robust GNR functionalization.

\section{Materials and Methods}

\section{Materials}


The Pd-diimine catalyst, $\left[(\mathrm{ArN}=\mathrm{C}(\mathrm{Me})-(\mathrm{Me}) \mathrm{C}=\mathrm{NAr}) \mathrm{Pd}\left(\mathrm{CH}_{3}\right)(\mathrm{NCMe})\right] \mathrm{SbF}_{6} \quad(\mathrm{Ar}=2,6-$ $\left.(i \operatorname{Pr})_{2} \mathrm{C}_{6} \mathrm{H}_{3}\right)$, was synthesized as per our earlier reports. ${ }^{48-55}$ The acrylate comonomers, including $\mathrm{AETA}^{+} \mathrm{BF}_{4}^{-}$, BIEA, and PMA, were synthesized according to the procedures reported in our earlier papers. ${ }^{49,55,71}$ BA (> 99\%; Aldrich) was passed through a column of basic alumina to remove the radical inhibitor. Deionized water was obtained from a Barnstead Nanopure II water purification system. OEGA $\left(M_{\mathrm{n}}=480 \mathrm{Da}\right), \mathrm{CTAB}(\geq 99 \%), \mathrm{NaBF}_{4}(98 \%), \mathrm{HAuCl}_{3} \cdot 3 \mathrm{H}_{2} \mathrm{O}$ (99.9\%), $\mathrm{AgNO}_{3}(99 \%), \mathrm{NaBH}_{4}(96 \%)$, Nile Red (technical grade), benzoyl peroxide (BPO, $\geq$ 98\%), $\mathrm{KOH}$ (90\%), $\mathrm{H}_{2} \mathrm{O}_{2}$ ( 50 wt.\% in water), 1-methyl-2-pyrrolidinone (NMP, 99\%), 1,4dioxane (99\%), acetone ( $\geq 99.5 \%$ ), methanol ( $\geq 99.5 \%$ ), and ethanol ( $\geq 99.5 \%)$ were obtained from Aldrich and used as received. $\mathrm{HCl}$ acid (certified ACS, Fisher Scientific), THF (HPLC grade), toluene (HPLC grade), chlorobenzene (HPLC grade), chloroform (HPLC grade), acetonitrile (HPLC grade), hexane (HPLC grade), ethyl acetate (certified ACS grade), NaCN (certified ACS grade; extremely toxic! handle by strictly following safety procedures), and $N, N$ dimethylformamide (DMF, certified ACS grade) were all received from Fisher Scientific and used without further purification.

\section{Synthesis of Quaternary Ammonium-Containing Hyperbranched Polyethylene Ionomers (HPEI0-HPEI6)}

All hyperbranched polyethylene ionomers containing the quaternary tetraalkylammonium cation with chloride counter ion and other desired functionalities (HPEI0-HPEI6) were synthesized by catalytic chain walking copolymerization of ethylene with $\mathrm{AETA}^{+} \mathrm{BF}_{4}^{-}$, along with other prescribed functional acrylate comonomers (BIEA, PMA, or OEGA), at room temperature. Representatively, the following is the procedure employed for the synthesis of HPEI3 in Table 1. A flame-dried Schlenk flask was purged with ethylene for 4 times, and then filled with ethylene to an absolute pressure of $1 \mathrm{~atm}$. Then the comonomer solution containing $\mathrm{AETA}^{+} \mathrm{BF}_{4}^{-}(0.49 \mathrm{~g}, 2$ mmol), PMA (0.086 g, $0.3 \mathrm{mmol})$, and BIEA (0.212 g, $0.8 \mathrm{mmol})$ in acetone $(9 \mathrm{~mL})$ was injected into the reactor. The polymerization was started upon the addition of the Pd-diimine catalyst solution ( $0.08 \mathrm{~g}$ or $0.1 \mathrm{mmol}$ in $1 \mathrm{~mL}$ of acetone). During the polymerization, ethylene pressure was maintained at 1 atm by continuous supply from a cylinder and the reaction temperature was maintained at room temperature. 
After $24 \mathrm{~h}$, the polymerization was stopped by venting the reactor, followed with the addition of $40 \mathrm{~mL}$ methanol. The resulting mixture was stirred overnight. Subsequently, the polymer precipitate was subject to three cycles of dissolution in THF and precipitation in methanol to remove residual comonomers and the diimine ligand. To remove the small $\mathrm{Pd}$ particles (from decomposed $\mathrm{Pd}$-diimine catalyst) trapped within the polymer, the polymer was dissolved in THF ( $2 \mathrm{~mL}$ ), and the mixture of $\mathrm{H}_{2} \mathrm{O}_{2} / \mathrm{HCl} / \mathrm{THF}(\mathrm{v} / \mathrm{v} / \mathrm{v}=1: 0.1: 10)$ was slowly added into the solution under stirring until the color of the polymer solution changed from black to light orange. Subsequently, the polymer was precipitated in methanol, followed with 3 more cycles of dissolution and precipitation. Finally, the precipitated polymer was dried under vacuum at $60{ }^{\circ} \mathrm{C}$ to give HPEI3 (0.87 g).

The resulting ionomers were all characterized with ${ }^{1} \mathrm{H}$ NMR spectroscopy. Figure S1 in ESI shows the ${ }^{1} \mathrm{H}$ NMR spectra of representative ionomers. In order for the characterization of their molecular weight and molecular weight distribution with gel permeation chromatography (GPC), the ionomers were hydrolyzed under basic conditions to cleave off the ionic ammonium groups. ${ }^{55}$ A typical procedure for the basic hydrolysis is as follows. A flask was added with $30 \mathrm{mg}$ of the ionomer dissolved in $5 \mathrm{~mL}$ of THF, along with $0.1 \mathrm{~g}$ of $\mathrm{KOH}$ dissolved in $5 \mathrm{~mL}$ of methanol. The mixture was refluxed for 3 days. The hydrolyzed polymer was precipitated out with methanol and was further washed with excessive methanol before drying at $60{ }^{\circ} \mathrm{C}$ under vacuum. Figure S3 in ESI shows the GPC elution curves of the hydrolyzed ionomers in THF as the mobile phase.

\section{Synthesis of the Poly(n-Butyl Acrylate) Ionomer (PBAI)}

The pyrene-labeled linear poly(n-butyl acrylate) ionomer containing the quaternary ammonium cations with $\mathrm{Cl}^{-}$as the counter anion was synthesized through radical polymerization. The polymerization procedure is as follows. To a pre-dried test tube were added $n$-butyl acrylate (1.28 g, $10 \mathrm{mmol}), \mathrm{AETA}^{+} \mathrm{BF}_{4}^{-}(45 \mathrm{mg}, 0.2 \mathrm{mmol})$, PMA (9 mg, $\left.0.03 \mathrm{mmol}\right)$, BPO (12 mg, 0.05

$\mathrm{mmol})$, and DMF (1 mL). The system was degassed via 4 freeze-pump-thaw cycles and was charged with $\mathrm{N}_{2}$. Then it was heated to $80{ }^{\circ} \mathrm{C}$ for $18 \mathrm{~h}$ to undergo radical copolymerization. After 
the polymerization, the reaction mixture was diluted with $3 \mathrm{~mL}$ of cold methanol and ca. $0.1 \mathrm{~g}$ of $\mathrm{NaCl}$ was added to exchange the $\mathrm{BF}_{4}^{-}$anion. Then the mixture was transferred to a dialysis tube. A large amount of methanol was used to wash off the solvent and unreacted comonomers. The resulting solution was dried under vacuum, rendering a light yellow ionomer (PBAI, $1.16 \mathrm{~g}$ ). Copolymer composition (determined with ${ }^{1} \mathrm{H}$ NMR, see Figure S2 in ESI): $\mathrm{AETA}^{+}, 1.95$ mol\%; PMA, $0.3 \mathrm{~mol} \%$.

\section{Synthesis of Gold Seed}

Gold seed was prepared by following the procedure reported by El-Sayed and Nikoobakht ${ }^{14}$ with minor modification. A cold aqueous solution of $0.01 \mathrm{M} \mathrm{NaBH}_{4}$ was freshly prepared. To an $\mathrm{HAuCl}_{4}$ solution $(20 \mathrm{~mL}, 0.5 \mathrm{mM})$ in $0.1 \mathrm{M} \mathrm{CTAB}$ was added $2.4 \mathrm{~mL}$ of the $\mathrm{NaBH}_{4}$ solution under rapid stirring. After continuous stirring for $10 \mathrm{~min}$, the seed solution was kept still at $25^{\circ} \mathrm{C}$ for $2 \mathrm{~h}$ before use.

\section{Synthesis of CTAB-Coated GNRs}

The two large-scale batches of CTAB-coated GNRs (long and short GNRs with similar rod diameter of $7.4 \mathrm{~nm}$ but different average aspect ratio of 6 and 4.7, respectively) used in this study were synthesized by scaling up the small-scale procedures reported by Zubarev and Vigderman, ${ }^{55}$ and El Sayed and Nikoobakht, ${ }^{24}$ respectively.

The following is the synthesis procedure for long GNRs. Fresh $\mathrm{AgNO}_{3}$ solution $(15 \mathrm{~mL}, 0.01 \mathrm{M})$ was added to an $\mathrm{HAuCl}_{4}$ solution $(750 \mathrm{~mL}, 0.5 \mathrm{mM})$ in $0.1 \mathrm{M} \mathrm{CTAB}$, followed by the addition of hydroquinone aqueous solution $(37.5 \mathrm{~mL}, 0.1 \mathrm{M})$. The mixed solution was stirred until it became clear. Subsequently, $12 \mathrm{~mL}$ of seed solution was added, and the growth solution was mixed thoroughly and allowed to age overnight. The resulting GNR dispersion was subject to two rounds of centrifugation (at 11,176 $\mathrm{g}$ for $20 \mathrm{~min}$ ) and resuspension in deionized water (800 $\mathrm{mL})$, and was then concentrated for subsequent use. The Au concentration $(2.0 \mathrm{mg} / \mathrm{mL})$ in the concentrated GNR dispersion was determined with flame Atomic Absorption spectroscopy. 
The following is the synthesis procedure for short GNRs. Typically, $11.3 \mathrm{~mL}$ of fresh prepared $\mathrm{AgNO}_{3}(0.01 \mathrm{M})$ was added into $750 \mathrm{~mL} \mathrm{HAuCl}{ }_{4}(0.5 \mathrm{mM})$ in $0.1 \mathrm{M} \mathrm{CTAB}$ solution. After mixing, $4.1 \mathrm{~mL}$ of fresh L-ascorbic acid $(0.1 \mathrm{M})$ was added into the system, followed by strong stirring until the orange color of the solution disappeared. Subsequently, $2 \mathrm{~mL}$ of seed solution was added, and the growth solution was mixed thoroughly and allowed to age overnight. The resulting GNR dispersion was subject to two rounds of centrifugation (at 11,176 g for $20 \mathrm{~min}$ ) and resuspension in deionized water $(800 \mathrm{~mL})$, and was then concentrated. The final $\mathrm{Au}$ concentration $(2.3 \mathrm{mg} / \mathrm{mL})$ in the concentrated GNR dispersion was determined with flame Atomic Absorption spectroscopy.

\section{Phase Transfer of CTAB-Coated GNRs from Aqueous Phase to Organic Phases with HPEI0}

A typical procedure for the phase transfer of the CTAB-coated long GNRs from aqueous phase to chlorobenzene organic phase with HPEI0 is as follows. To a $25 \mathrm{ml}$ glass vial was added $2 \mathrm{~mL}$ aqueous solution of long GNRs (Au concentration $=2.0 \mathrm{mg} / \mathrm{mL}$ ), along with $2 \mathrm{~mL}$ of chlorobenzene containing $5 \mathrm{mg}$ of HPEI0 as the organic phase. The biphase mixture was stirred overnight. Subsequently, $1 \mathrm{~g}$ of $\mathrm{NaCl}$ salt dissolved in $2 \mathrm{~mL}$ of water was then added under gentle stirring. The addition of $\mathrm{NaCl}$ led to the swift phase transfer of the GNRs to the organic phase. The organic phase containing the GNRs was then diluted by 50 times for UV-vis analysis.

\section{Preparation of Ionomer-Modified GNRs by Direct Dropping Method}

Representatively, a typical procedure for the synthesis of ionomer-modified GNRs (I-GNR3-3 in Table 2) by ligand exchange of CTAB-coated short GNRs with HPEI3 is as follows. An aqueous dispersion $(2 \mathrm{~mL}$, with $[\mathrm{Au}]=2.3 \mathrm{mg} / \mathrm{mL})$ of short GNRs was added slowly over 10 min into a THF solution of HPEI3 (4 mg in $40 \mathrm{~mL}$ ) under vigorous stirring. The mixture was further stirred for $30 \mathrm{~min}$ and then centrifuged at 7,184 $\mathrm{g}$ for $15 \mathrm{~min}$ to precipitate out the ionomer-modified GNRs. To remove residual CTAB and excessive ionomer, the precipitated GNRs were dispersed in $20 \mathrm{~mL}$ of THF, followed with the addition of $20 \mathrm{~mL}$ of ethanol. The dispersion was subject to subsequent centrifugation at 11,176 g (20 min). After another round of wash with THF/ethanol 
by centrifugation, the nanorods were further centrifuged at 11,176 $\mathrm{g}$ (20 $\mathrm{min}$ ) for 10 rounds with fresh THF to further remove residual ionomer and/or CTAB and also to examine the stability of the modified nanorods under multiple rounds of centrifugation. During the process, the precipitated GNRs and the corresponding supernatant solutions were monitored with fluorescence spectroscopy in order to determine the ionomer mass content in the ionomermodified GNRs.

Other ionomer-modified GNRs were similarly prepared with minor variations in their conditions as follows. The ionomer-modified GNRs, from I-GNR1 to I-GNR5 except I-GNR2 and I-GNR33 , were prepared by adding $0.2 \mathrm{~mL}$ of aqueous dispersion (containing Au at $2.3 \mathrm{mg} / \mathrm{mL}$ ) of short GNRs into $4 \mathrm{~mL}$ of ionomer solution in THF at the prescribed ionomer concentration ([ionomer $]_{0}$, see Table 2), followed with purification to remove residual CTAB and/or ionomer. I-GNR2 was prepared by adding $5 \mathrm{~mL}$ of aqueous dispersion of short GNRs (containing Au at $2.3 \mathrm{mg} / \mathrm{mL}$ ) into $100 \mathrm{~mL}$ of HPEI 2 solution in THF ([HPEI2 $\left.]_{0}=0.4 \mathrm{mg} / \mathrm{mL}\right)$, followed with purification by centrifugation cycles. I-GNR6 was prepared by adding $13 \mathrm{~mL}$ of aqueous dispersion of long GNRs $([\mathrm{Au}]=2.0 \mathrm{mg} / \mathrm{mL})$ into $100 \mathrm{~mL}$ of HPEI6 solution in methanol $\left([\mathrm{HPEI} 6]_{0}=1 \mathrm{mg} / \mathrm{mL}\right)$, followed purification by multiple rounds of centrifugation.

\section{Cyanide Treatment of I-GNR2}

In order to identify the surface ligands present in the ionomer-modified GNRs with ${ }^{1} \mathrm{H}$ NMR spectroscopy, the gold core of the GNRs was dissolved with $\mathrm{NaCN}$ to release the surface-bound organic materials. ${ }^{22,23,35}$ A typical procedure is as follows. To a dispersion of the HPEI2-modified nanorods, I-GNR2, in $\mathrm{CH}_{2} \mathrm{Cl}_{2}$ (11 mg of I-GNR2 containing $3.4 \mathrm{mg}$ of HPEI2 in $1 \mathrm{~mL} \mathrm{CH}_{2} \mathrm{Cl}_{2}$ ) was added $1 \mathrm{~mL}$ of $\mathrm{NaCN}$ aqueous solution (20 mg of $\mathrm{NaCN}$ and $20 \mathrm{mg}$ of $\mathrm{NaOH}$ in $1 \mathrm{~mL} \mathrm{D}_{2} \mathrm{O}$ ). The mixture was stirred overnight. Subsequently, the aqueous $\mathrm{D}_{2} \mathrm{O}$ phase was directly characterized with ${ }^{1} \mathrm{H}$ NMR (10,000 scans) and was found not to contain any organic species. The organic phase was dried by evaporation and the resulting white solid was re-dissolved in $\mathrm{CDCl}_{3} /$ methanol $(\mathrm{v} / \mathrm{v}=2: 1$; total $0.6 \mathrm{~mL})$ for the characterization with ${ }^{1} \mathrm{H}$ NMR spectroscopy (10,000 scans). 


\section{Nile Red Encapsulation within HPEI6 and I-GNR6 in Aqueous Dispersions}

A Nile Red mother dispersion in water at a concentration of $0.8 \mathrm{mg} / \mathrm{mL}$ was first prepared by dispersing $8 \mathrm{mg}$ of Nile Red in $10 \mathrm{~mL}$ of deionized water, followed with sonication for $48 \mathrm{~h}$. In the case with amphiphilic HPEI6 as the carrier, $0.1 \mathrm{~mL}$ of the freshly prepared Nile Red mother dispersion was added into $5 \mathrm{~mL}$ of HPEI6 dispersion in water (concentration: $0.5 \mathrm{mg} / \mathrm{mL}$ ). The mixture was sonicated at room temperature to reach equilibrium. The dispersion was then filtrated with a $0.45 \mu \mathrm{m}$ syringe filter to remove the unencapsulated Nile Red. The filtrate was analyzed with fluorescence spectroscopy (emission spectrum obtained at the excitation wavelength $\lambda_{\mathrm{ex}}=535 \mathrm{~nm}$ and excitation spectrum obtained at the emission wavelength $\lambda_{\mathrm{em}}=601$ $\mathrm{nm})$. With reference to a calibration curve generated based on sodium dodecyl sulfateencapsulated Nile Red (see Figure S10 in ESI), the concentration of Nile Red encapsulated within HPEI6 at this condition was quantified to be $0.23 \mathrm{mg} / \mathrm{L}$ or $0.72 \mu \mathrm{M}$, with $1.4 \%$ of fed Nile Red encapsulated. Nile Red loading capacity of HPEI6 is thus $0.046 \mathrm{wt} \%$ (mass of encapsulated Nile Red relative to mass of the carrier HPEI6).

A similar procedure was also employed for the encapsulation with I-GNR6 as the carrier. The mixture of the freshly prepared Nile Red mother dispersion $(0.1 \mathrm{~mL})$ and I-GNR6 dispersion in water $(5 \mathrm{~mL}$ at concentration of $5.7 \mathrm{mg} / \mathrm{mL})$ was sonicated to reach equilibrium. After standing overnight, the supernatant was carefully collected and analyzed with fluorescence spectroscopy. Quantification with reference to the calibration curve rendered a concentration of $0.16 \mathrm{mg} / \mathrm{L}$ or $0.51 \mu \mathrm{M}$ for the encapsulated Nile Red in the dispersion. Nile Red encapsulation capacity with IGNR6 is thus $0.003 \mathrm{wt} \%$ (or $0.09 \mu \mathrm{mol} / \mathrm{g}$ ) of the mass of I-GNR6 or $0.024 \mathrm{wt} \%$ (or $0.8 \mu \mathrm{mol} / \mathrm{g}$ ) of the mass of HPEI6 present in I-GNR6.

\section{Synthesis of PBAI-Modified GNRs}

The synthesis of PBAI-modified GNRs is similar to that of polyethylene ionomer-modified GNRs. A PBAI solution $([\mathrm{PBAI}]=0.2 \mathrm{mg} / \mathrm{mL})$ was prepared by dissolving $1 \mathrm{mg}$ of PBAI in 5 $\mathrm{mL}$ of THF. Then $0.2 \mathrm{~mL}$ of aqueous dispersion of long GNRs ([Au] $=2 \mathrm{mg} / \mathrm{mL}$ ) was added dropwise to the PBAI solution under vigorous stirring. The mixture was stirred for another 30 
min. Then the resulting PBAI-modified GNRs were washed twice with THF/ethanol mixture and then another three times with THF by centrifugation (11,176 g for $20 \mathrm{~min})$. The resulting dispersion of PBAI-modified GNRs in THF was then characterized with UV-vis and fluorescence spectroscopy. As per the quantification with fluorescence spectroscopy, the modified GNRs have an $m_{\mathrm{PBAI}} / m_{\mathrm{Au}}$ ratio of 0.24 , with $10 \%$ of fed PBAI being adsorbed. To prepare dispersions in other solvents (acetone, ethanol), the above dispersion in THF was concentrated by centrifugation and re-dispersed in the corresponding solvent, followed with one more cycle of centrifugation to remove residual THF and the addition of the corresponding pure solvent. UV-vis characterization was subsequently undertaken on the resulting dispersions.

\section{Instrumentation and Characterization}

Proton nuclear magnetic resonance $\left({ }^{1} \mathrm{H}\right.$ NMR) spectra of all polymers were obtained on a Varian Gemini 2000 spectrometer $(200 \mathrm{MHz})$ at ambient temperature with $\mathrm{CDCl}_{3}$ as solvent. Atomic absorption spectroscopy was performed on an AAnalyst 440 spectrometer (Perkin Elmer) equipped with an Au element lamp (Max. $60 \mathrm{~mA}$, Perkin Elmer). The data was collected by winLab32 software (Perkin Elmer). The blank solution was 1\% aqua regia solution in water. For all the analyses, a calibration curve was first established with Au standard solutions with $[\mathrm{Au}]$ in the range of $0.25-10 \mathrm{mg} / \mathrm{L}$.

GPC characterization of the hydrolyzed ionomers was carried out on a Polymer Laboratories PLGPC220 system equipped with a differential refractive index (DRI) detector (from Polymer Laboratories) and a four-bridge capillary viscosity detector (from Polymer Laboratories). See our earlier paper on details for the GPC characterization. ${ }^{55} \mathrm{UV}$-vis spectroscopy was performed on a Thermoscientific Genesys 10S UV-vis spectrophotometer. Fluorescence spectroscopy was performed on an Olis RSM 1000 Desa rapid-scanning monochromator spectrophotometer system. DLS characterization was carried out on a Brookhaven NanoBrook Omni Instrument at $25^{\circ} \mathrm{C}$. TEM images were collected on a JEOL 2010F field emission electron microscope or a JEOL $2100 \mathrm{LaB}_{6}$ microscope at an operating voltage of $200 \mathrm{kV}$. Thermogravimetric analysis was undertaken on a TA Instruments Q50 TGA in a nitrogen atmosphere from 100 to $800{ }^{\circ} \mathrm{C}$ with a heating rate of $10^{\circ} \mathrm{C} / \mathrm{min}$. 


\section{Electronic Supplementary Information (ESI) Available}

${ }^{1} \mathrm{H}$ NMR spectra of representative ionomers (Figures S1 and S2), GPC curves of the hydrolyzed ionomers (Figure S3), Tables for additional GPC and NMR characterization results of the ionomers (Table S1), UV-vis spectra from phase transfer control experiments (Figure S4), fluorescence spectra of pyrene-containing ionomers and modified GNRs (Figure S5), fluorescence calibration curves (Figure S6), examples on the determination of ionomer mass content in the modified GNRs (Table S2), TGA curves for I-GNR2 and HPEI2 (Figure S7), UVvis spectra of I-GNR3-3 in other solvents (Figure S8), Nile Red fluorescence spectra and calibration curve (Figures S9 and S10). See DOI: 10.1029/xxxx.

\section{Acknowledgement}

The financial support from Canada Research Chair (\#220084) is greatly appreciated. ZD thanks the Ontario Ministry of Economic Development and Innovation for awarding a postdoctoral fellowship. PX thanks the Ontario Ministry of Training, Colleges and Universities and Laurentian University for awarding two Ontario Graduate Scholarships. The authors also thank Prof. Catherine J. Murphy and Joshua G. Hinman at the University of Illinois at UrbanaChampaign for assistance in the TEM characterization and helpful discussion.

\section{References and Notes}

1. C. J. Murphy, T. K. Sau; A. M. Gole, C. J. Orendorff, J. Gao, L. Gou, S. E. Hunyadi and T. Li, J. Phy. Chem. B, 2005, 109, 13857-13870.

2. J. Pérez-Juste, I. Pastoriza-Santos, L. M. Liz-Marzán and P. Mulvaney, Coord. Chem. Rev., 2005, 249, 1870-1901.

3. X. Huang, S. Neretina and M. A. El-Sayed, Adv. Mater., 2009, 21, 4880-4910.

4. E. C. Dreaden, M. A. Mackey, X. Huang, B. Kang and M. A. El-Sayed, Chem. Soc. Rev., 2011, 40, 3391-3404. 
5. A. M. Alkilany, L. B. Thompson, S. P. Boulos, P. N. Sisco and C. J. Murphy, Adv. Drug Deliv. Rev., 2012, 64, 190-199.

6. L. Vigderman, B. P. Khanal and E. R. Zubarev, Adv. Mater., 2012, 24, 4811-4841.

7. E. C. Dreaden, A. M. Alkilany, X. Huang, C. J. Murphy and M. A. El-Sayed, Chem. Soc. Rev., 2012, 41, 2740-2779.

8. A. M. Alkilany, S. E. Lohse and C. J. Murphy, Acc. Chem. Res., 2013, 46, 650-661.

9. H. Chen, L. Shao, Q. Li and J. Wang, Chem. Soc. Rev., 2013, 42, 2679-2724.

10. M. J. A. Hore and R. J. Composto, Macromolecules, 2014, 47, 875-887.

11. S. E. Lohse and C. J. Murphy, Chem. Mater., 2013, 25, 1250-1261.

12. N. R. Jana, L. Gearheart and C. J. Murphy, Adv. Mater., 2001, 13, 1389-1393.

13. N. R. Jana, L. Gearheart and C. J. Murphy, J. Phys. Chem. B, 2001, 105, 4065-4067.

14. B. Nikoobakht and M. A. El-Sayed, Chem. Mater., 2003, 15, 1957-1962.

15. B. Nikoobakht and M. A. El-Sayed, Langmuir, 2001, 17, 6368-6374.

16. H. Takahashi, Y. Niidome, T. Niidome, K. Kaneko, H. Kawasaki and S. Yamada, Langmuir, 2006, 22, 2-5.

17. A. M. Alkilany, P. K. Nagaria, C. R. Hexel, T. J. Shaw, C. J. Murphy and M. D. Wyatt, Small, 2009, 5, 701-708.

18. A. S. D. S. Indrasekara, R. C. Wadams and L. Fabris, Part. Part. Sys. Charact., 2014, 31, 819-838.

19. E. Locatelli, I. Monaco and M. C. Franchini, RSC Adv., 2015, 5, 21681-21699.

20. T. Niidome, M. Yamagata, Y. Okamoto, Y. Akiyama, H. Takahashi, T. Kawano, Y. Katayama and Y. Niidome, J. Control. Release, 2006, 114, 343-347.

21. M. Liu, W.-C. Law, A. Kopwitthaya, X. Liu, M. T. Swihart and P. N. Prasad, Chem. Commun., 2013, 49, 9350-9352.

22. C. Kinnear, H. Dietsch, M. J. D. Clift, C. Endes, B. Rothen-Rutishauser and A. Petri-Fink, Angew. Chem. Int. Ed., 2013, 52, 1934-1938.

23. L. Vigderman, P. Manna and E. R. Zubarev, Angew. Chem. Int. Ed., 2012, 51, 636-641.

24. Q. Dai, J. Coutts, J. Zou and Q. Huo, Chem. Commun., 2008, 2858-2860.

25. D. Gentili and M. C. Franchini, Chem. Commun., 2009, 5874-5876.

26. B. Thierry, J. Ng, T. Krieg and H. J. Griesser, Chem. Commun., 2009, 1724-1726.

27. A. Wijaya and K. Hamad-Schifferli, Langmuir, 2008, 24, 9966-9969. 
28. A. Wijaya, S. B. Schaffer, I. G. Pallares and K. Hamad-Schifferli, ACS Nano, 2009, 3, 8086.

29. H. Liao and J. H. Hafner, Chem. Mater., 2005, 17, 4636-4641.

30. A. Gole and C. J. Murphy, Chem. Mater., 2005, 17, 1325-1330.

31. A. P. Leonov, J. Zheng, J. D. Clogston, S. T. Stern, A. K. Patri and A. Wei, ACS Nano, 2008, 2, 2481-2488.

32. A. M. Alkilany, L. B. Thompson and C. J. Murphy, ACS Appl. Mater. Interface, 2010, 2, 3417-3421.

33. H.-C. Huang, S. Barua, D. B. Kay and K. Rege, ACS Nano, 2009, 3, 2941-2952.

34. D. Pissuwan and T. Niidome, Nanoscale, 2015, 7, 59-65.

35. C. J. Orendorff, T. M. Alam, D. Y. Sasaki, B. C. Bunker and J. A. Voigt, ACS Nano, 2009, 3, 971-983.

36. S. E. Lee, D. Y. Sasaki, T. D. Perroud, D. Yoo, K. D. Patel and L. P. Lee, J. Am. Chem. Soc., 2009, 131, 14066-14074.

37. A. M. Alkilany, P. K. Nagaria, M. D. Wyatt and C. J. Murphy, Langmuir, 2010, 26, 93289333.

38. I. Pastoriza-Santos, J. Pérez-Juste and L. M. Liz-Marzán, Chem. Mater., 2006, 18, 24652467.

39. Y. Zhang, J. Qian, D. Wang, Y. Wang and S. He, Angew. Chem. Int. Ed., 2013, 52, 11481151.

40. Z. Zhang, L. Wang, J. Wang, X. Jiang, X. Li, Z. Hu, Y. Ji, X. Wu and C. Chen, Adv. Mater., 2012, 24, 1418-1423.

41. A. Eisenberg and J.-S. Kim, Introduction to Ionomers; John Wiley \& Sons: New York, 1998.

42. M. I. Burguete, E. García-Verdugo, S. V. Luis and J. A. Restrepo, Phys. Chem. Chem. Phys., 2011, 13, 14831-14838.

43. J. Restrepo, P. Lozano, M. I. Burguete, E. García-Verdugo and S. V. Luis, Catal. Today, $2015,255,97-101$.

44. J. Restrepo, R. Porcar, P. Lozano, M. I. Burguete, E. García-Verdugo and S. V. Luis, ACS Catal., 2015, 5, 4743-4750.

45. Z. Dong and Z. Ye, Polym. Chem., 2012, 3, 286-301. 
46. Z. Ye, L. Xu, Z. Dong and P. Xiang, Chem. Commun., 2013, 49, 6235-6255.

47. The bromoisobutyryl initiating sites present in HPEI2-HPEI5 are highly active in initiating atom-transfer radical polymerization of various monomers (see Ref. 49). Though not used yet in the work, this functionality enables further grafting of functional polymer chains onto these ionomers, which may provide another methodology to add additional functionalities onto the resulting modified GNRs.

48. J. Wang, Z. Ye and H. Joly, Macromolecules, 2007, 40, 6150-6163.

49. K. Zhang, J. Wang, R. Subramanian, Z. Ye, J. Lu and Q. Yu, Macromol. Rapid Commun., 2007, 28, 2185-2191.

50. J. Wang, K. Zhang and Z. Ye, Macromolecules, 2008, 41, 2290-2293.

51. J. Ye, Z. Ye and S. Zhu, Polymer, 2008, 49, 3382-3392.

52. S. Morgan, Z. Ye, K. Zhang and R. Subramanian, Macromol. Chem. Phys., 2008, 209, 2232-2240.

53. P. Xiang and Z. Ye, Macromol. Rapid Commun., 2010, 31, 1083-1089.

54. P. Liu, Z. Ye, W. J. Wang and B. G. Li, Macromolecules, 2012, 46, 72-82.

55. P. Xiang and Z. Ye, Macromolecules, 2015, 48, 6096-6107.

56. L. Vigderman and E. R. Zubarev, Chem. Mater., 2013, 25, 1450-1457.

57. H. Chen, L. Shao, K. C. Woo, T. Ming, H.-Q. Lin and J. Wang, J. Phys. Chem. C, 2009, 113, 17691-17697.

58. Y. Khalavka, J. Becker and C. Sönnichsen, J. Am. Chem. Soc., 2009, 131, 1871-1875.

59. H. Chen, X. Kou, Z. Yang, W. Ni and J. Wang, Langmuir, 2008, 24, 5233-5237.

60. J. Yang, J.-C. Wu, Y.-C. Wu, J.-K. Wang and C.-C. Chen, Chem. Phys. Lett., 2005, 416, 215-219.

61. M. Karg, I. Pastoriza-Santos, J. Pérez-Juste, T. Hellweg and L. M. Liz-Marzán, Small, 2007, 3, 1222-1229.

62. M. Liu, W.-C. Law, A. Kopwitthaya, X. Liu, M. T. Swihart and P. N. Prasad, Chem. Comm., 2013, 49, 9350-9352.

63. M. G. Soliman, B. Pelaz, W. J. Parak and P. del Pino, Chem. Mater., 2015, 27, 990-997.

64. J. M. El Khoury, X. Zhou, L. Qu, L. Dai, A. Urbas and Q. Li, Chem. Commun., 2009, 21092111. 
65. A. M. Alkilany, A. I. B. Yaseen, J. Park, J. R. Eller and C. J. Murphy, RSC Adv., 2014, 4, $52676-52679$.

66. G.-T. Wei, Z. Yang, C.-Y. Lee, H.-Y. Yang and C. R. C. Wang, J. Am. Chem. Soc., 2004, 126, 5036-5037.

67. A. Wijaya and K. Hamad-Schifferli, Langmuir, 2008, 24, 9966-9969.

68. N. S. Abadeer, M. R. Brennan, W. L. Wilson and C. J. Murphy, ACS Nano, 2014, 8, 83928406.

69. B. Rostro-Kohanloo, L. R. Bickford, C. M. Payne, E. S. Day, L. J. E. Anderson, M. Zhong, S. Lee, K. M. Mayer, T. Zal, L. Adam, C. P. N. Dinney, R. A. Drezek, J. L. West and J. H. Hafner, Nanotechnology, 2009, 20, 434005.

70. G. Chen and Z. Guan, J. Am. Chem. Soc., 2004, 126, 2662-2663.

71. L. Xu, Z. Ye, S. Siemann and Z. Gu, Polymer, 2014, 55, 3120-3129. 


\section{Supporting Information}

for

\section{Efficient, Robust Surface Functionalization and Stabilization of Gold Nanorods with Quaternary Ammonium-Containing Ionomers as Multidentate Macromolecular Ligands}

Zhongmin Dong, Peng Xiang, Lingqi Huang, and Zhibin Ye*

Bharti School of Engineering, Laurentian University, 935 Ramsey Lake Road, Sudbury, Ontario P3E 2C6, Canada

* Corresponding author; email: zye@laurentian.ca 


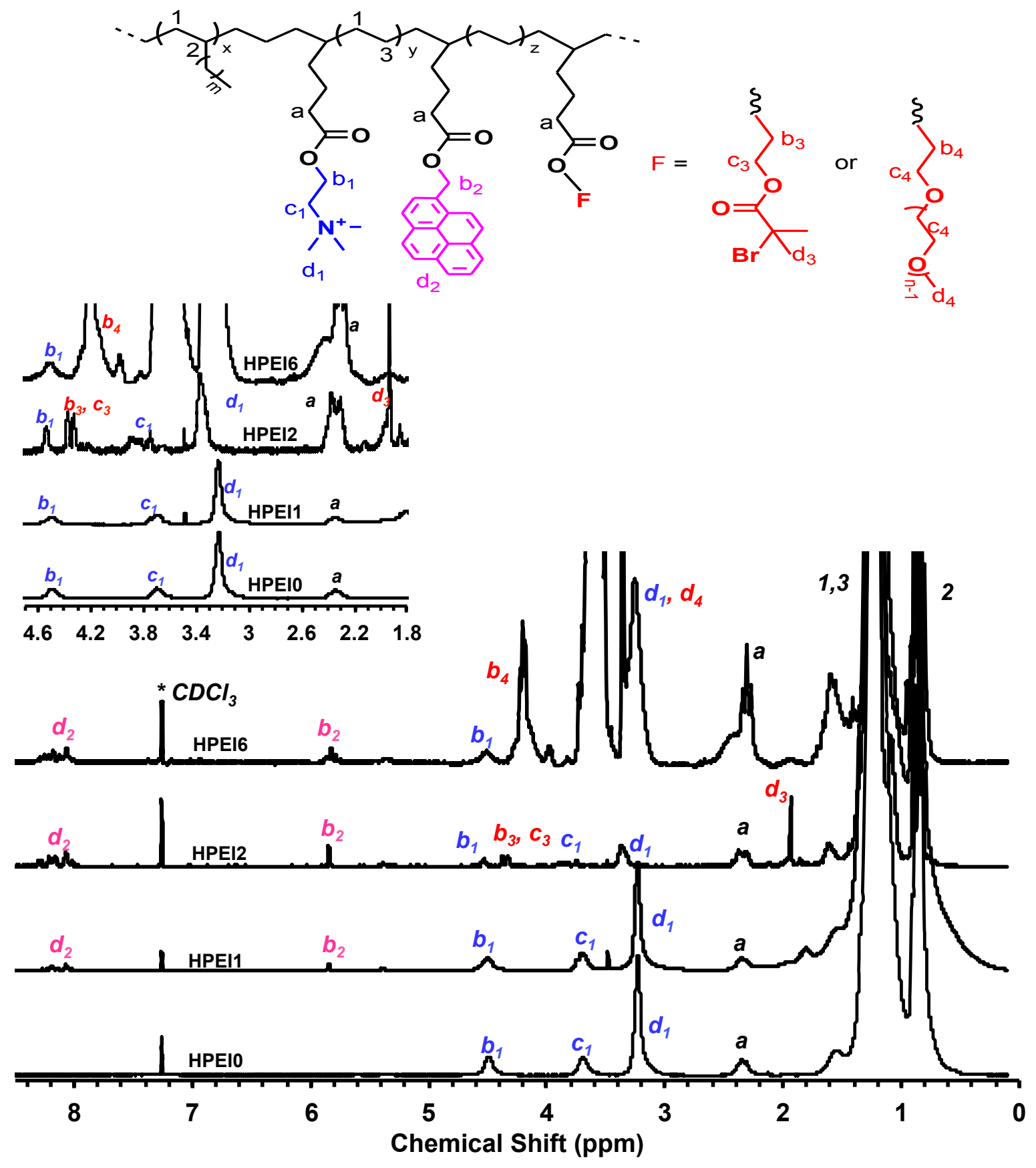

Figure S1. ${ }^{1} \mathrm{H}$ NMR spectra (in $\mathrm{CDCl}_{3}$ ) of representative hyperbranched polyethylene ionomers (HPEI0-HPEI2 and HPEI6) containing different functionalities. 

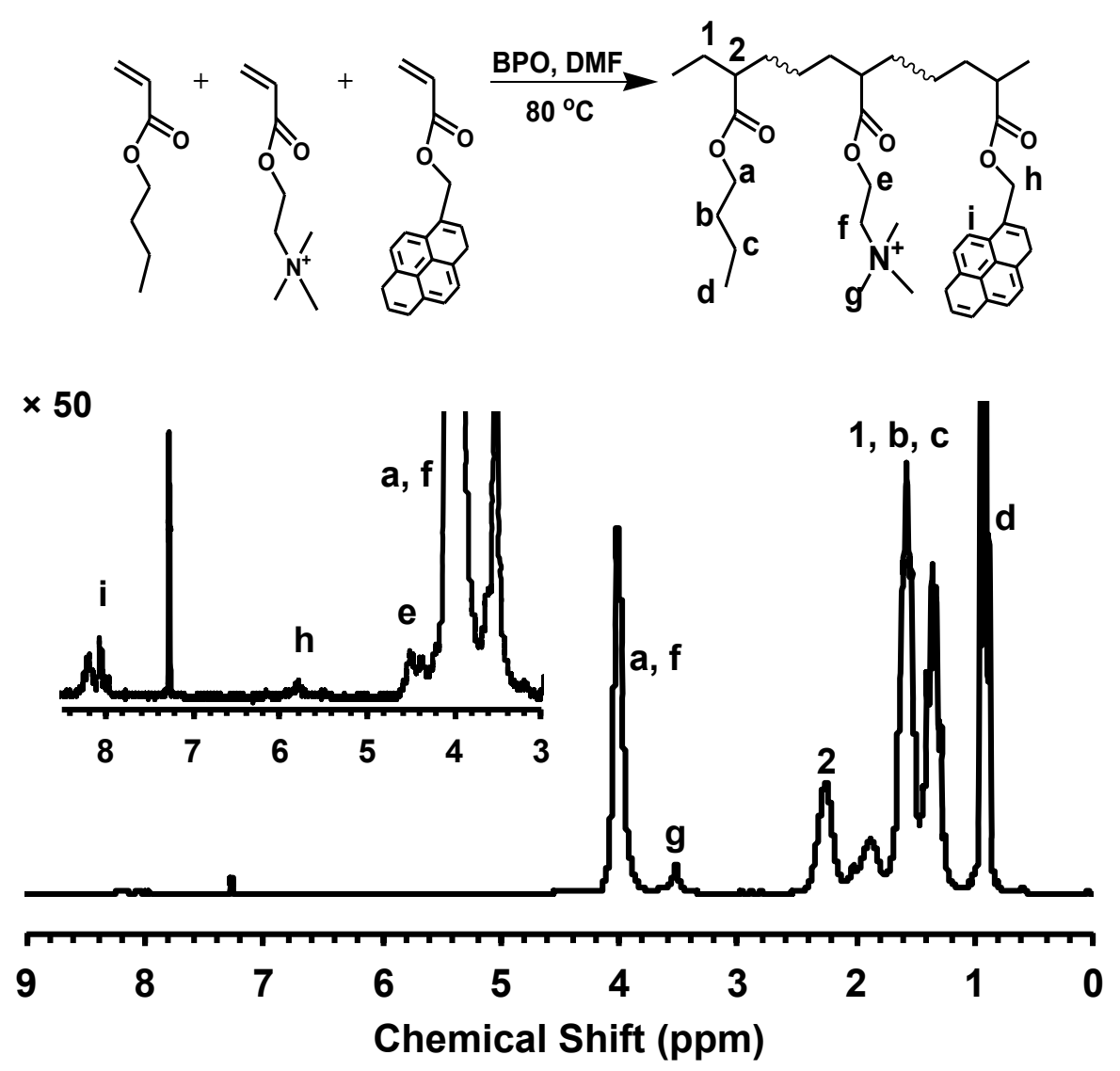

Figure S2. ${ }^{1} \mathrm{H}$ NMR spectrum of pyrene-labelled quaternary ammonium-containing poly $(n$-butyl acrylate) ionomers, PBAI. 


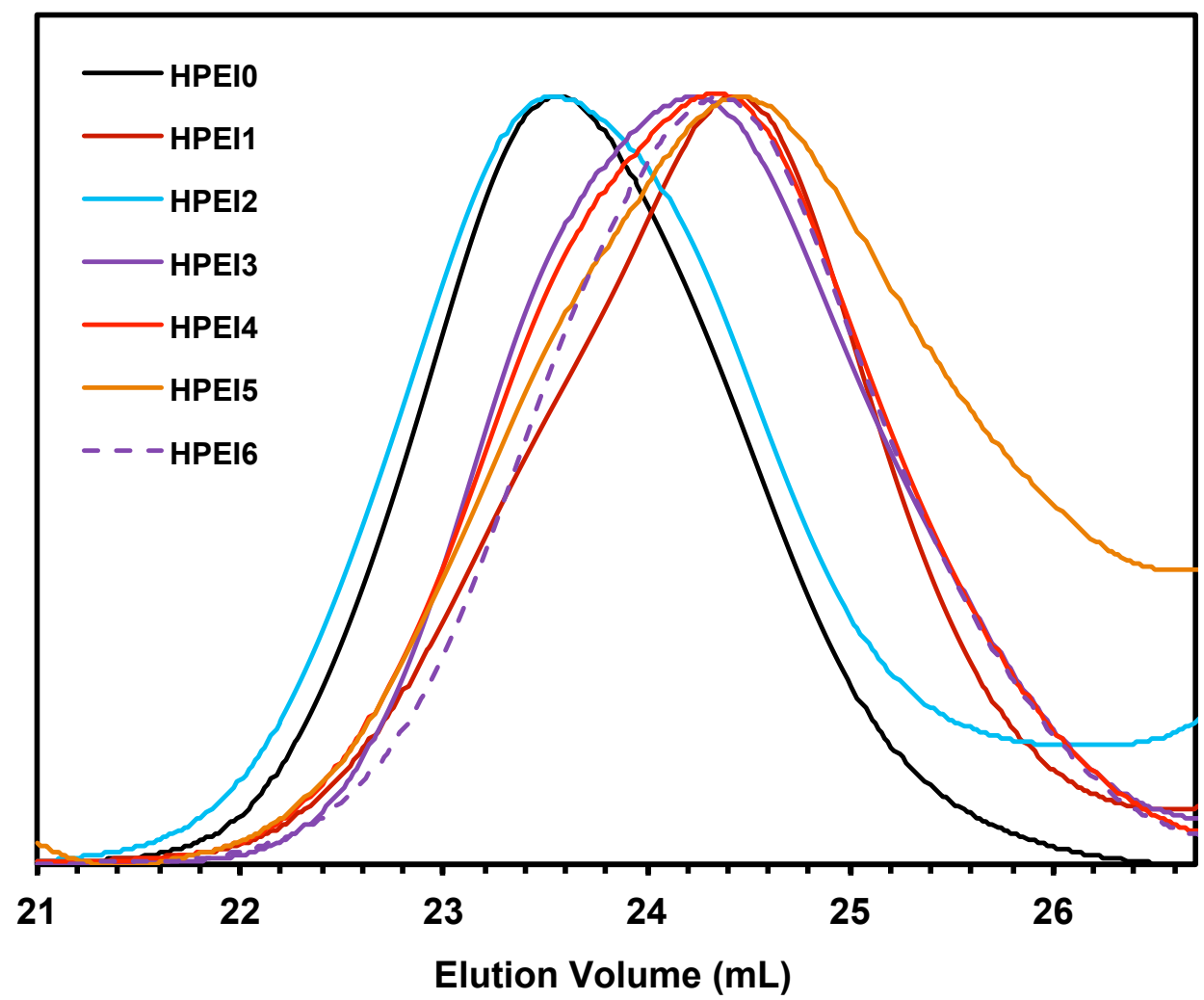

Figure S3. GPC elution curves (recorded with the DRI detector) of the hydrolyzed hyperbranched polyethylene ionomers. THF as the mobile phase at $1 \mathrm{~mL} / \mathrm{min}$ and $33{ }^{\circ} \mathrm{C}$. 
Table S1. Branching density, molecular weight data, and dilute solution properties of the hydrolyzed hyperbranched polyethylene ionomers. ${ }^{a}$

\begin{tabular}{lllllll}
\hline Ionomer & $\begin{array}{l}\text { Branch } \\
\text { density }^{b} \\
(\text { per 1000 C) }\end{array}$ & $\begin{array}{l}M_{\mathrm{n}}{ }^{c} \\
(\mathrm{kDa})\end{array}$ & $\begin{array}{l}M_{\mathrm{w}}{ }^{c} \\
(\mathrm{kDa})\end{array}$ & $\mathrm{PDI}^{c}$ & $\begin{array}{l}\mathrm{M}-\mathrm{H} \\
\alpha^{d}\end{array}$ & $\begin{array}{l}\eta_{\mathrm{n}}{ }^{e} \\
(\mathrm{~mL} / \mathrm{g})\end{array}$ \\
\hline HPEI0 $^{e}$ & 83 & 13.3 & 18.8 & 1.42 & 0.35 & 12.1 \\
HPEI1 $^{f}$ & 96 & 12.2 & 16.3 & 1.33 & 0.36 & 10.5 \\
HPEI2 & 83 & 38.3 & 43.3 & 1.13 & 0.34 & 12.9 \\
HPEI3 & 86 & 4.2 & 8.8 & 2.08 & 0.29 & 10.3 \\
HPEI4 & 100 & 3.4 & 7.8 & 2.29 & 0.25 & 10.4 \\
HPEI5 & 87 & 8.7 & 11.0 & 1.26 & -0.05 & 9.1 \\
HPEI6 & 96 & 3.1 & 8.3 & 2.69 & 0.13 & 12.3 \\
\hline
\end{tabular}

${ }^{a}$ See Table 1 for details on the synthesis of the ionomers. ${ }^{b}$ Branching density of ethylene sequences of the ionomers determined from their ${ }^{1} \mathrm{H}$ NMR spectra. ${ }^{c}$ Number- and weightaverage molecular weight, and polydispersity index of the hydrolyzed ionomers. ${ }^{d}$ MarkHouwink $\alpha$ constant of the hydrolyzed ionomers. ${ }^{e}$ Number-average intrinsic viscosity of the hydrolyzed ionomers. 


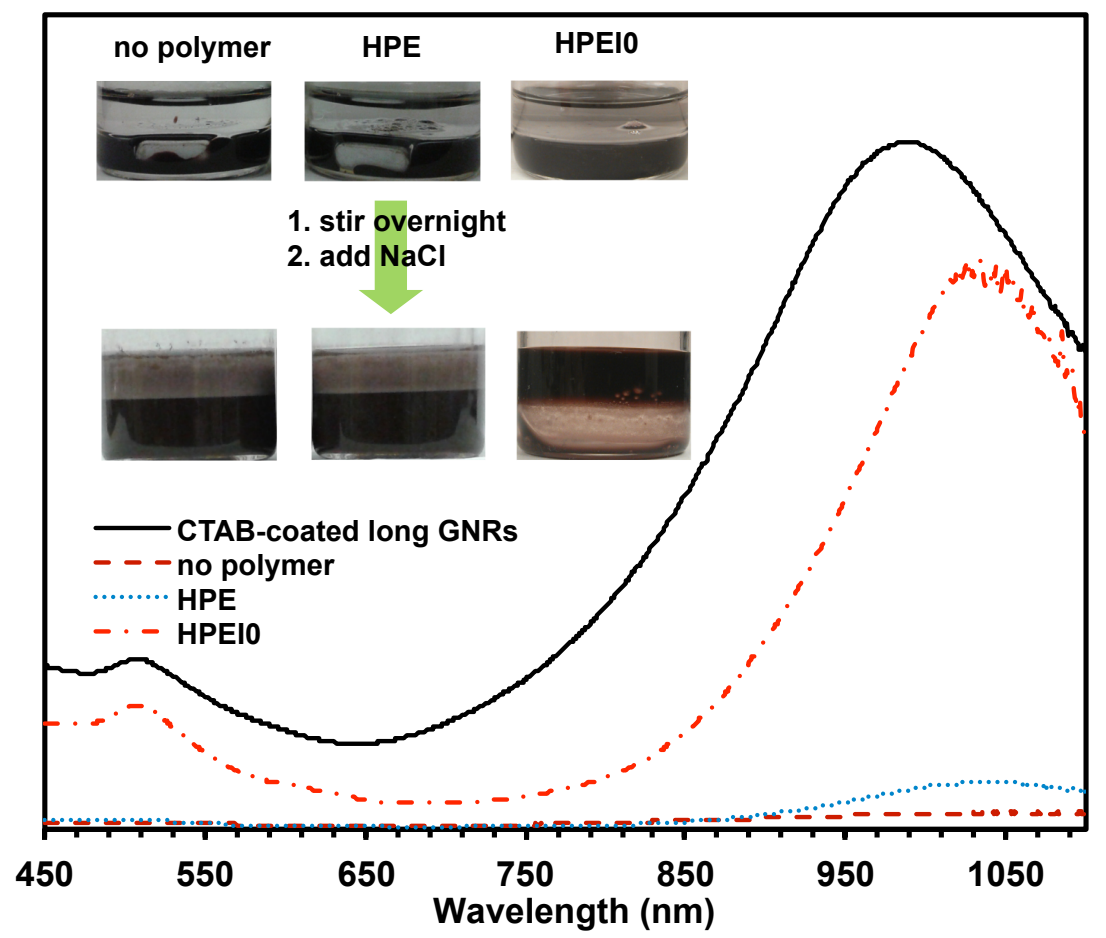

Figure S4. Control experiments showing the unsuccessful phase transfer of CTAB-coated long GNRs from aqueous phase to organic toluene phase in the case with no polymer or with nonionic hyperbranched polyethylene (HPE): UV-vis spectra of the resulting toluene phases (after the same dilution). The inset shows the photographs of the biphase mixtures before and after the process. The photographs and spectrum achieved with the use of HPEI0 are also included for comparison. 


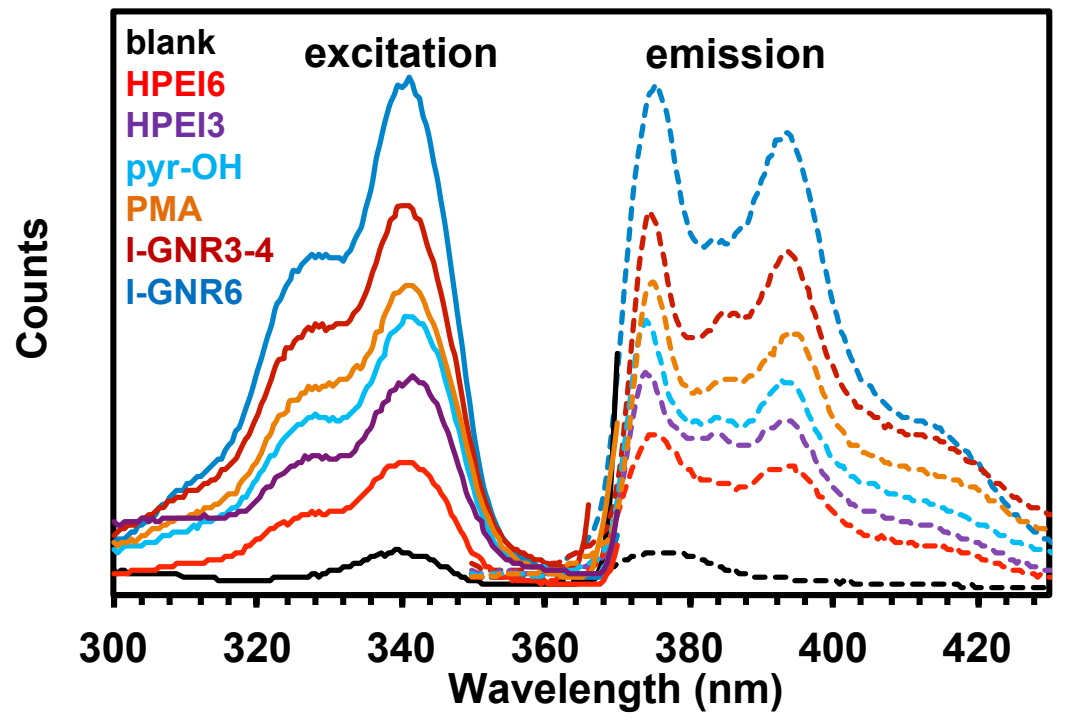

Figure S5. Fluorescent emission (em) and excitation (ex) spectra of pyrene-containing precursors, pyrene methanol (pyr-OH) and pyrenylmethyl acrylate (PMA), and representative pyrene-functionalized ionomers (HPEI3 and HPEI6) and corresponding ionomer-modified GNRs (I-GNR3-4 and I-GNR6). All the spectra were taken in THF as solvent. The emission spectra were collected at the excitation wavelength $\lambda_{\mathrm{ex}}=341.5 \mathrm{~nm}$; the excitation spectra were collected at the emission wavelength $\lambda_{\mathrm{em}}=374.5 \mathrm{~nm}$. 

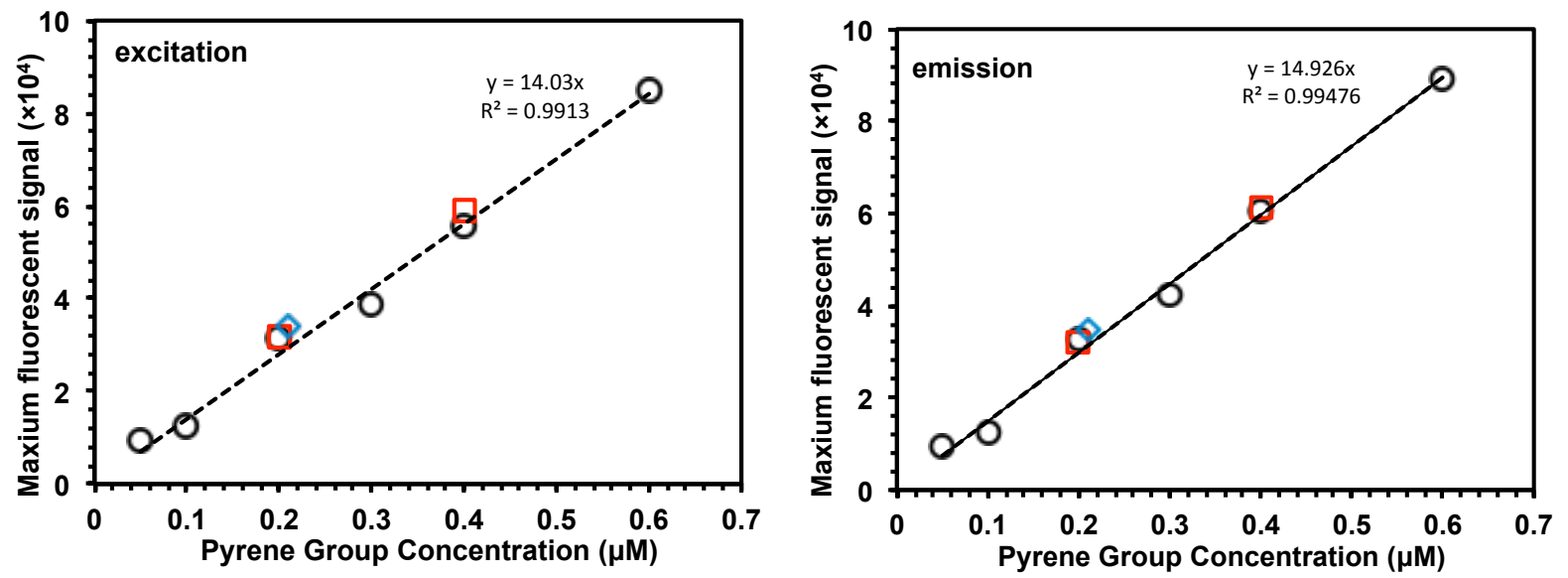

Figure S6. The fluorescence emission and excitation calibration curves (correlating the fluorescence intensity at $341.5 \mathrm{~nm}$ and $374.5 \mathrm{~nm}$, respectively, vs. pyrene group concentration in THF) generated with pyrenylmethyl acrylate (PMA) as the concentration standard $(\circ$, concentration: $0.05,0.1,0.2,0.3,0.4$, and $0.6 \mu \mathrm{mol} / \mathrm{L})$. The fluorescence signals of with other pyrene-containing compounds/polyethylene ionomers with predetermined concentrations ( $\square$ for pyrene methanol with concentrations of 0.2 and $0.4 \mu \mathrm{mol} / \mathrm{L}$; $\diamond$ for HPEI2 with polymer concentration of $1 \mathrm{mg} / \mathrm{L}$ and pyrene group concentration of around $0.21 \mu \mathrm{mol} / \mathrm{L}$ ) are also included, which fit the calibration curves well. 
Table S2. Determination of ionomer content in representative ionomer-coated GNRs (I-GNR3-4 and I-GNR6) and the corresponding supernatant solutions by fluorescence quantification during the centrifugation purification procedure.

\begin{tabular}{|c|c|c|c|c|c|c|}
\hline sample & $\begin{array}{l}\text { ionomer } \\
\text { feed mass } \\
\quad(\mathrm{mg})\end{array}$ & $\begin{array}{c}\mathrm{Au} \\
\text { mass, } \\
m_{\mathrm{Au}}, \\
(\mathrm{mg})\end{array}$ & $\begin{array}{c}\begin{array}{c}\text { Solution } \\
\text { volume }^{c}\end{array} \\
(\mathrm{~mL})\end{array}$ & $\begin{array}{l}\text { Pyrene } \\
\text { group } \\
\text { conc. }^{d} \\
(\mu \mathrm{M})\end{array}$ & $\begin{array}{l}\text { ionomer } \\
\text { mass, } \\
m_{\text {ionomer }} \\
(\mathrm{mg})\end{array}$ & $\begin{array}{c}m_{\text {ionomer }} / m_{\mathrm{Au}} \\
(\mathrm{mg} / \mathrm{mg})\end{array}$ \\
\hline I-GNR3-4 $^{e}$ & 0.80 & 0.46 & 40 & 0.14 & 0.08 & 0.17 \\
\hline Supernat. 1 & & & 200 & 0.24 & 0.66 & \\
\hline Supernat. 2 & & & 20 & 0.12 & 0.03 & \\
\hline Supernat. 3 & & & 20 & 0.05 & 0.01 & \\
\hline total & & & & & $0.78^{g}$ & \\
\hline I-GNR6 $^{g}$ & 1.0 & 0.1 & 8 & 0.08 & 0.014 & 0.14 \\
\hline Supernat. 1 & & & 200 & 0.16 & 0.72 & \\
\hline Supernat. 2 & & & 50 & 0.03 & 0.034 & \\
\hline Supernat. 3 & & & 5 & 0.10 & 0.011 & \\
\hline total & & & & & $0.78^{g}$ & \\
\hline
\end{tabular}

${ }^{a}$ The initial feed mass of ionomer to perform the ligand exchange with CTAB-coated GNRs. For other details, see Table 2 in the article on the preparation the two ionomer-coated GNRs ${ }^{b}$ The mass of $\mathrm{Au}$ in the resulting ionomer-modified GNRs. ${ }^{c}$ The volume of the resulting ionomermodifiedd GNR dispersions or the supernatant solutions collected during the purification of ionomer-modified GNRs by centrifugation. ${ }^{d}$ The pyrene concentration in the ionomer-modified GNR dispersions or the corresponding supernatant solutions quantified through fluorescence analysis. Negligible pyrene concentration was found in the supernatant solutions obtained after the first 3 centrifugations. ${ }^{e}$ The ionomer mass in the dispersions of ionomer-modified GNRs or in the supernatant solutions. ${ }^{f}$ The mass ratio of ionomer to Au in the ionomer-modified GNRs. ${ }^{g}$ The total ionomer mass in the ionomer-modified GNRs and the supernatant solutions. 


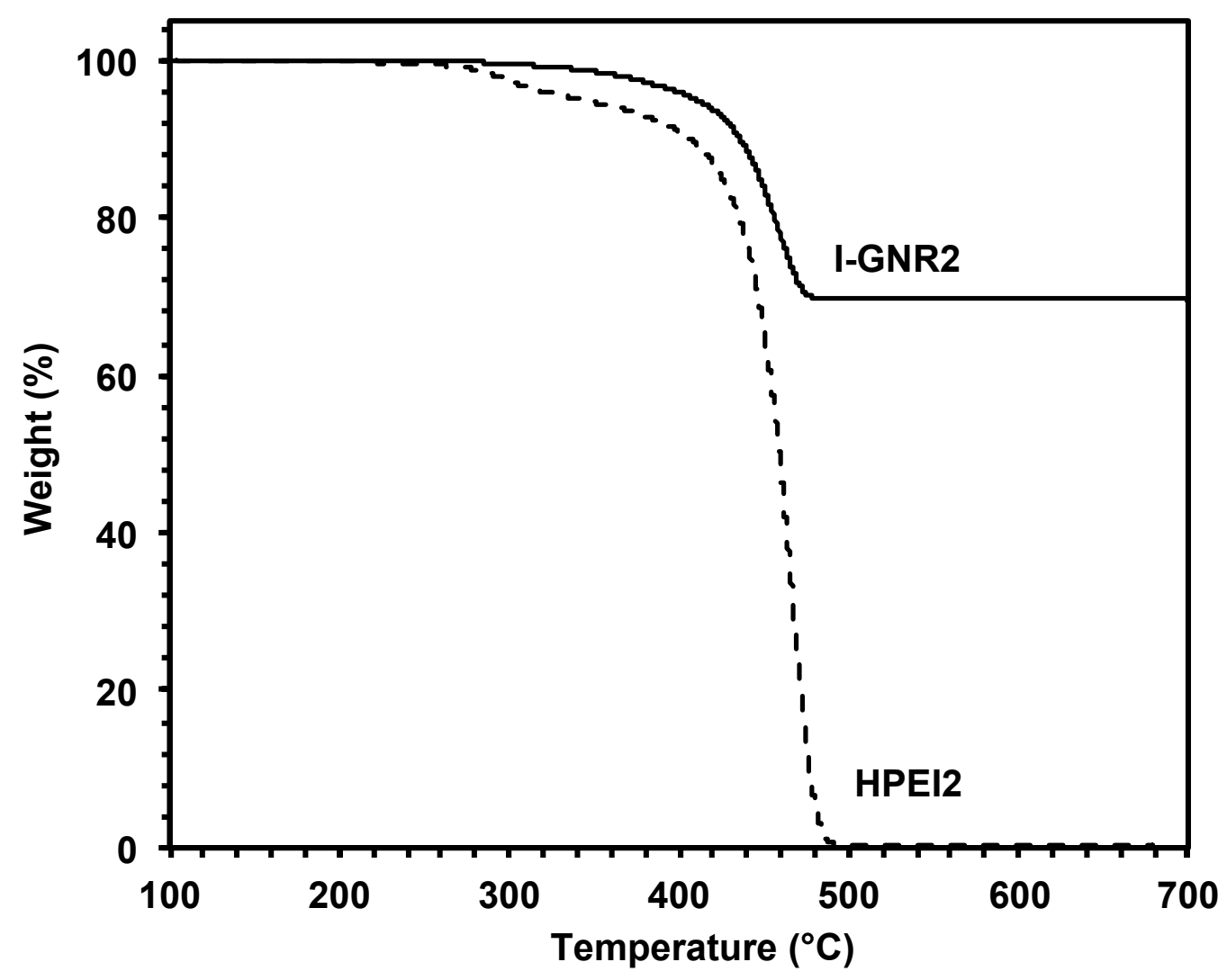

Figure S7. TGA curves of pure HPEI2 and the HPEI2-modified GNRs, I-GNR2. TGA was undertaken in a nitrogen atmosphere with a heating rate of $10{ }^{\circ} \mathrm{C} / \mathrm{min}$. As per the TGA results, IGNR2 has a HPEI2 content of $30.5 \mathrm{wt} \%$, which agrees well with the value of $31.0 \mathrm{wt} \%$ determined by fluorescence measurement. 


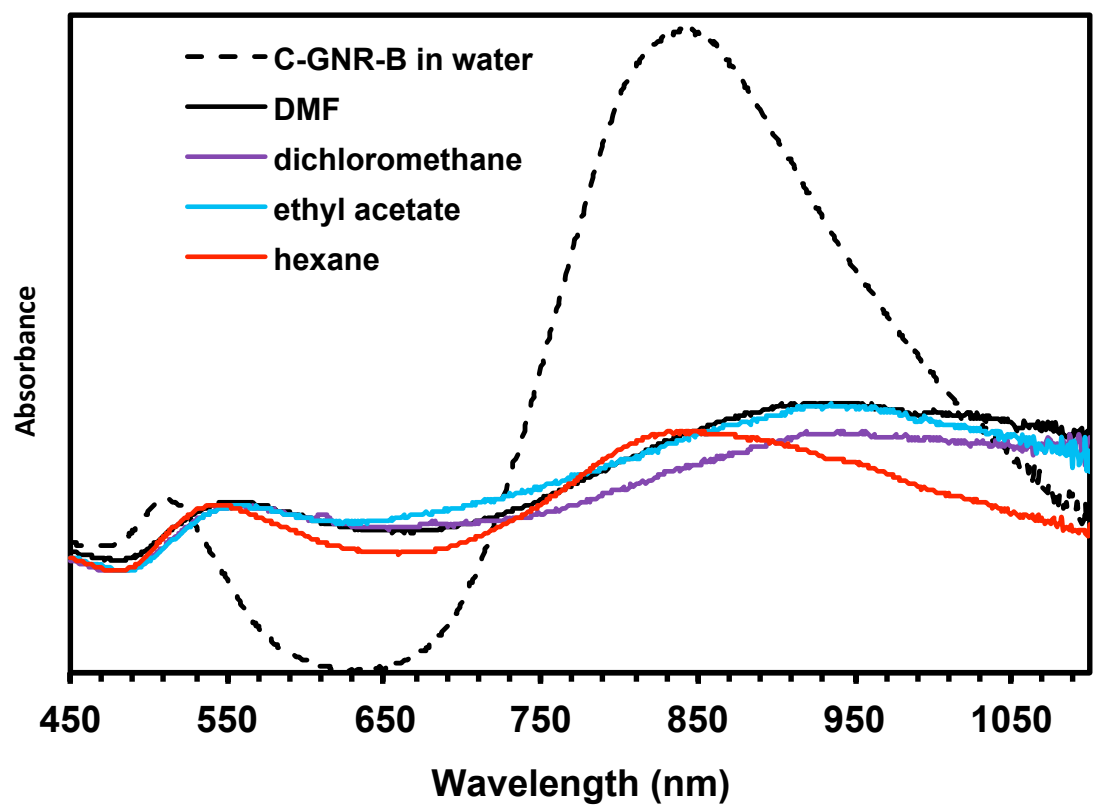

Figure S8. UV-vis spectra of I-GNR3-3 in DMF, dichloromethane, ethyl acetate, and hexane. The dispersions were prepared by adding a small volume of THF solution of I-GNR3-3 into the corresponding solvent (volume ratio $=1: 9$ ), followed with one round of centrifugation and the resuspension in the corresponding pure solvent. 


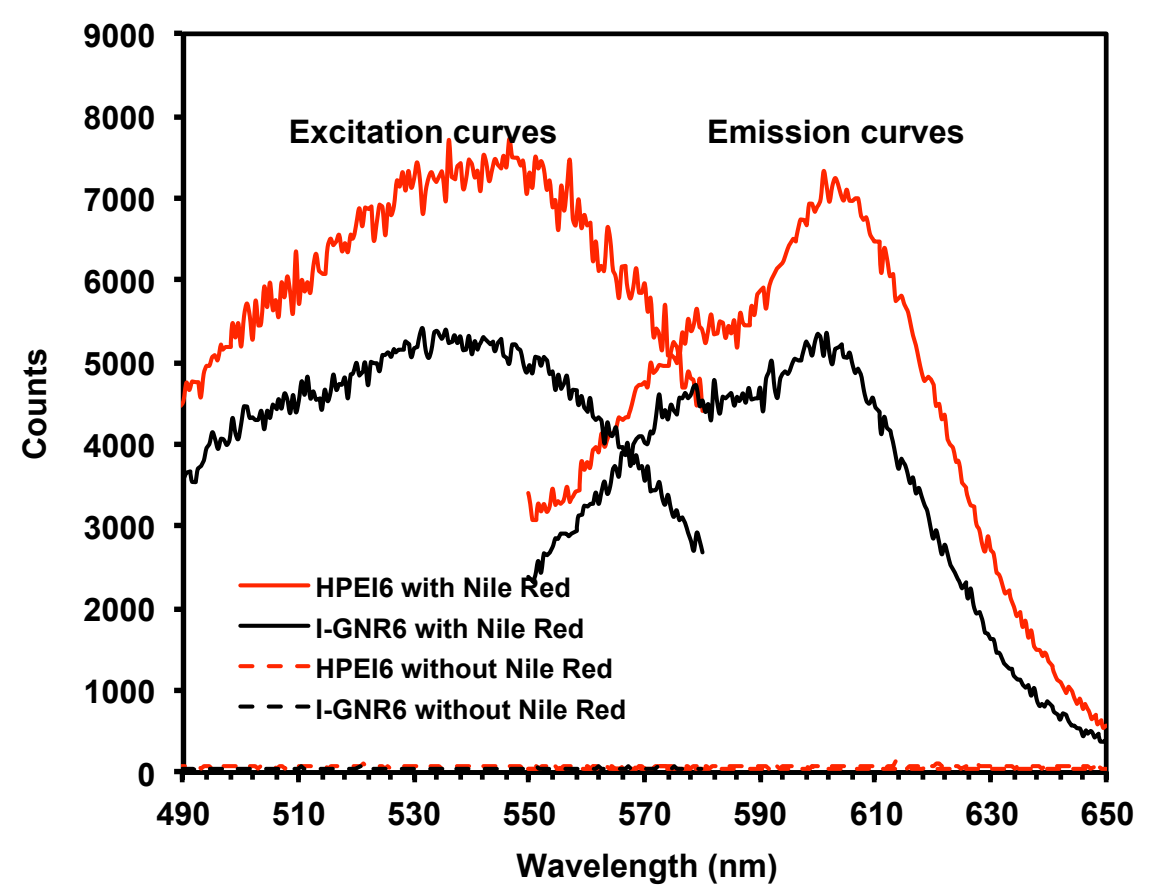

Figure S9. Fluorescence spectra of HPEI6 (concentration: $0.5 \mathrm{mg} / \mathrm{mL}$ ) and I-GNR6 (concentration: $5.7 \mathrm{mg} / \mathrm{mL}$ ) encapsulating Nile Red with the excitation spectra taken at emission wavelength $\lambda_{\mathrm{em}}=601 \mathrm{~nm}$ and emission spectra taken with excitation wavelength $\lambda_{\mathrm{em}}=535 \mathrm{~nm}$. The spectra of HPEI6 and I-GNR6 without containing Nile Red are also included for comparison. 


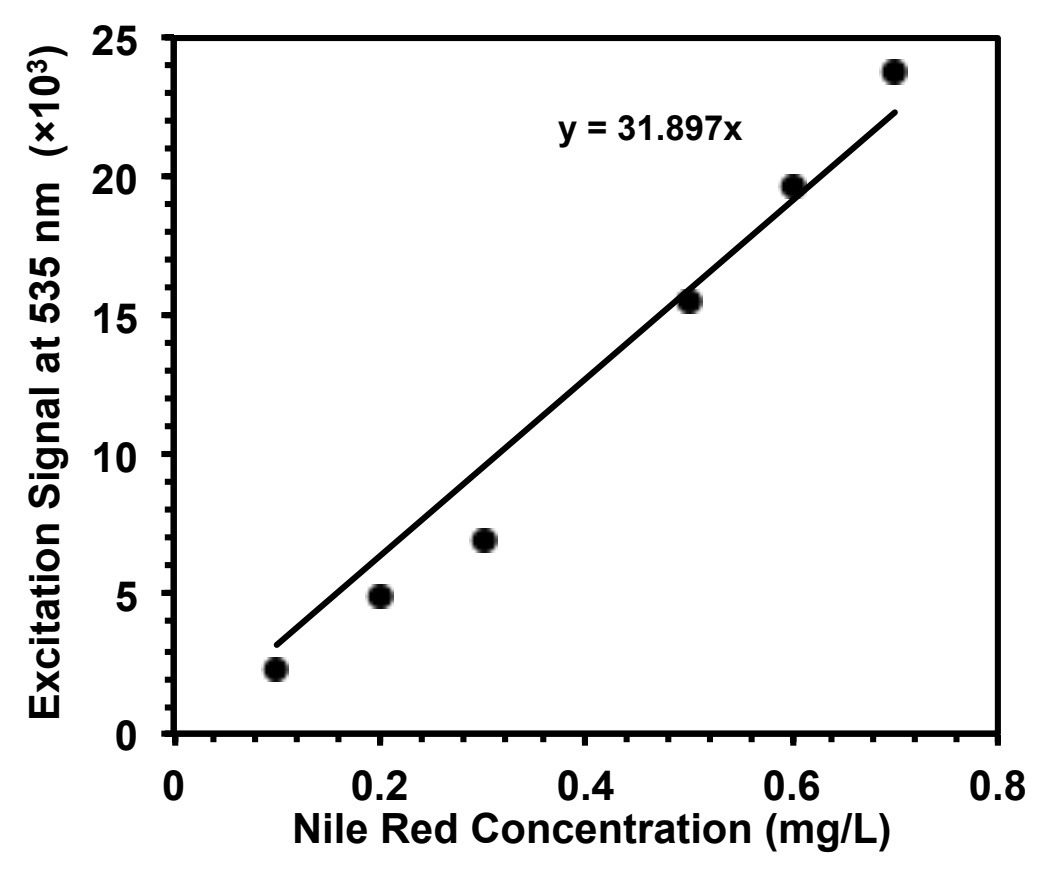

Figure S10. The calibration curve correlating the fluorescent excitation signal at $535 \mathrm{~nm}\left(\lambda_{\mathrm{em}}=\right.$ $601 \mathrm{~nm}$ ) with the concentration of Nile Red encapsulated within sodium dodecyl sulfate (SDS) in aqueous dispersion. Standard dispersions containing different concentration $(0.1,0.2,0.3,0.5$, 0.6 , and $0.7 \mathrm{mg} / \mathrm{L}$ ) of Nile Red encapsulated within SDS (a fixed concentration of $30 \mathrm{mg} / \mathrm{mL}$ in the dispersion) were prepared by referring to the work by Guan et al. (J. Am. Chem. Soc. 2004, 126, 2662-2663). Fluorescent excitation spectra of these dispersions were measured at $\lambda_{\mathrm{em}}=601$ $\mathrm{nm}$, which show maximum intensity at $535 \mathrm{~nm}$. 